\title{
Power and Channel Allocation for Non- Orthogonal Multiple Access in 5G Systems: Tractability and Computation
}

\author{
Lei Lei, Di Yuan, Chin Keong Ho and Sumei Sun \\ Journal Article
}

\section{Tweet}

N.B.: When citing this work, cite the original article.

C2016 IEEE. Personal use of this material is permitted. However, permission to reprint/republish this material for advertising or promotional purposes or for creating new collective works for resale or redistribution to servers or lists, or to reuse any copyrighted component of this work in other works must be obtained from the IEEE.

Lei Lei, Di Yuan, Chin Keong Ho and Sumei Sun, Power and Channel Allocation for NonOrthogonal Multiple Access in 5G Systems: Tractability and Computation, IEEE TRANSACTIONS ON WIRELESS COMMUNICATIONS, 2016. 15(12), pp.8580-8594.

http://dx.doi.org/10.1109/TWC.2016.2616310

Postprint available at: Linköping University Electronic Press

http://urn.kb.se/resolve?urn=urn:nbn:se:liu:diva-134309

LINKÖPINGS

UNIVERSITET 


\title{
Power and Channel Allocation for Non-orthogonal Multiple Access in 5G Systems: Tractability and Computation
}

\author{
Lei Lei ${ }^{1}$, Di Yuan ${ }^{1}$, Chin Keong $\mathrm{Ho}^{2}$, and Sumei Sun $^{2}$ \\ ${ }^{1}$ Department of Science and Technology, Linköping University, Sweden \\ ${ }^{2}$ Institute for Infocomm Research $\left(\mathrm{I}^{2} \mathrm{R}\right), \mathrm{A}^{*} \mathrm{STAR}$, Singapore \\ Emails: \{lei.lei;di.yuan@liu.se\},\{hock; sunsm\}@i2r.a-star.edu.sg
}

\begin{abstract}
A promising multi-user access scheme, nonorthogonal multiple access (NOMA) with successive interference cancellation (SIC), is currently under consideration for 5G systems. NOMA allows more than one user to simultaneously access the same frequency-time resource and separates multi-user signals by SIC. These render resource optimization in NOMA different from orthogonal multiple access. We provide theoretical insights and algorithmic solutions to jointly optimize power and channel allocation in NOMA. We mathematically formulate NOMA resource allocation problems, and characterize and analyze the problems' tractability under a range of constraints and utility functions. For tractable cases, we provide polynomial-time solutions for global optimality. For intractable cases, we prove the NP-hardness and propose an algorithmic framework combining Lagrangian duality and dynamic programming (LDDP) to deliver near-optimal solutions. To gauge the performance of the solutions, we also provide optimality bounds on the global optimum. Numerical results demonstrate that the proposed algorithmic solution can significantly improve the system performance in both throughput and fairness over orthogonal multiple access as well as over a previous NOMA resource allocation scheme.
\end{abstract}

Index Terms-Non-orthogonal multiple access, resource allocation, successive interference cancellation, 5G.

\section{INTRODUCTION}

Orthogonal multi-user access (OMA) techniques are used in $4 \mathrm{G}$ long term evolution (LTE) and LTE-Advanced (LTEA) networks, e.g., orthogonal frequency division multiple access (OFDMA) for downlink and single-carrier frequency division multiple access (SC-FDMA) for uplink [1], [2]. In OMA, within a cell, each user has exclusive access to the allocated resource blocks. Thus, each subchannel or subcarrier can only be utilized by at most one user in every time slot. OMA avoids intra-cell interference, and enables single-user detection/decoding and simple receiver design. However, by its nature, orthogonal channel access is becoming a limiting factor of spectrum efficiency.

In the coming decade, mobile data traffic is expected to grow thousand-fold [3], [4]. Accordingly, the network capacity must dramatically increase for $5 \mathrm{G}$ systems. Capacity scaling for $5 \mathrm{G}$ is enabled by a range of techniques and schemes, e.g., cell densification, utilization of unlicensed spectrum, and advanced radio access schemes. New multi-user access schemes have been investigated as potential alternatives to OFDMA and
SC-FDMA [3]-[5]. A promising scheme is the so-called nonorthogonal multiple access (NOMA) with successive interference cancellation (SIC) [6]. Unlike interference-avoidance multiple access schemes, e.g., OFDMA, multiple users in NOMA can be assigned to the same frequency-time resource so as to improve spectrum efficiency [6]. On one hand, this results in intra-cell interference among the multiplexed users. On the other hand, some of the interfering signals in NOMA can be eliminated by multi-user detection (MUD) with SIC at the receiver side. To enable this process, more advanced receiver design and interference management techniques are considered for $5 \mathrm{G}$ networks [4].

\section{A. Related Works}

From an information theory perspective, under the assumption of simultaneous multi-user transmission via superposition coding with SIC, capacity region and duality analysis have been studied in [7]. The authors of [8] have provided an analysis of implementing interference cancellation in cellular systems. Towards future $5 \mathrm{G}$ communication systems, some candidate access schemes are under investigation in recent research activities, e.g., sparse code multiple access (SCMA) [3], and NOMA [6].

In [9], the authors studied the capacity region for NOMA. In [10], by assuming predefined user groups for each subchannel, a heuristic algorithm for NOMA power allocation in downlink has been proposed, and system-level simulations have been conducted. In [11], the authors address various implementation issues of NOMA. The authors in [12] considered a sum-rate utility maximization problem for dynamic NOMA resource allocation. In [13], outage performance of NOMA has been evaluated. The authors derived the ergodic sum rate and outage probability to demonstrate the superior performance of NOMA with fixed power allocation. In [14], fairness considerations and a max-min fairness problem for NOMA have been addressed. The fairness in NOMA can be improved via using and adapting the so-called power allocation coefficients. For uplink NOMA, the authors in [15] provided a suboptimal algorithm to solve an uplink scheduling problem with fixed transmission power. In [16], a weighted multi-user scheduling scheme is proposed to balance the total throughput and the cell-edge 
user throughput. In [17], the authors proposed a greedy-based algorithm to improve the throughput in uplink NOMA. In [18], the authors studied and evaluated user grouping/pairing strategies in NOMA. It has been shown that, from the outage probability perspective, it is preferable to multiplex users of large gain difference on the same subcarrier. In [19], the authors address subcarrier allocation and power assignment in downlink NOMA, with the objective of balancing the throughout with the number of scheduled users. The solution approach uses matching theory, by first assuming equal power split and applying a user-subchannel matching algorithm that converges to a stable matching, followed by a water-filling phase for power allocation. In [20], a monotonic optimization method is developed for NOMA subcarrier and power allocation. The method potentially approaches the global optimum, at the cost of high complexity in the number of users per subcarrier. We remark that there are other setups of SIC than that considered in NOMA. An example is the interference channel in which common information is transmitted for partial interference cancellation, for which Etkin et al. [21] provided an analysis of the resulting capacity region and trade-off from an information theory perspective.

We note that in some studies of NOMA (e.g., [10], [14], [18]), the aspect of subcarrier or subchannel allocation was simplified or not addressed. However, to fully reach the potential of NOMA, user-subchannel allocation is of high significance due to fading. Assuming uniform subchannels or using fixed rules for subchannel allocation may result in significant performance loss. The importance of subchannel allocation is evidenced by the growing interest in explicitly taking this aspect into account in some recent works [12], [19], [20].

Apart from investigation of NOMA performance in cellular networks, from an optimization perspective, the complexity and tractability analysis of NOMA resource allocation is of significance. Here, tractability for an optimization problem refers to whether or not any polynomial-time algorithm can be expected to find the global optimum [1]. Tractability results for resource allocation in OMA and interference channels have been investigated in a few existing works, e.g., [1], [22], [23] for OFDMA, [2] for SC-FDMA, and [23], [24] for interference channel. For NOMA, to the best of our knowledge, no such study is available in the existing literature.

\section{B. Contributions}

In spite of the existing literature of performance evaluation for NOMA, there is lack of a systematic approach for NOMA resource allocation from a mathematical optimization point of view. The existing resource allocation approaches for NOMA are typically carried out with fixed power allocation [13], [15], [17], predefined user set for subchannels [10], or parameter tuning to improve performance, e.g., updating power allocation coefficients [14]. Moreover, compared with OMA, NOMA allows multi-user sharing on the same subchannel, thus provides an extra dimension to influence the performance in throughput and fairness. However, how to balance these two key performance aspects in power and channel allocation is largely not yet studied in the literature. In addition, little is known on the computational complexity and tractability of NOMA resource allocation.

In this paper, the solutions of joint channel and power allocation for NOMA are subject to systematic optimization, rather than using heuristics or ad-hoc methods. To this end, we formulate, analyze, and solve the power and channel optimization problem for downlink NOMA systems, taking into account practical considerations of fairness and SIC. We present the following contributions. First, for maximum weightedsum-rate (WSR) and sum-rate (SR) utilities, we formulate the joint power and channel allocation problems (JPCAP) mathematically. Second, we prove the NP-hardness of JPCAP with WSR and SR utilities. Third, we identify tractable cases for JPCAP and provide the tractability analysis. Fourth, we propose an algorithmic framework based on Lagrangian duality and dynamic programming to facilitate problem solving. Unlike previous works, our approach contributes to delivering near-optimal solutions, as well as performance bounds on global optimum to demonstrate the quality of our near-optimal solutions. We use numerical results to illustrate the significant performance improvement of the proposed algorithm over existing NOMA and OFDMA schemes.

Our work extends previous study of user grouping in NOMA. In [18], the number of users to be multiplexed on a subcarrier is fixed, and performance evaluation consists of rule-based multiplexing policies. In our case, for each subcarrier, the number of users and their composition are both output from solving an optimization problem. Later in Section VII, results of optimized subcarrier assignment and user grouping will be presented for analysis. The current paper extends our previous study [12] in several dimensions. The extensions consist of the consideration of the WSR utility metric, a significant amount of additional theoretical analysis of problem tractability, the development of the performance bound on global optimality, as well as the consideration of user fairness in performance evaluation.

The rest of the paper is organized as follows. Section II gives the system models for single-carrier and multi-carrier NOMA. Section III formulates JPCAP for WSR utility and provides complexity analysis. Section IV analyzes the tractability for special cases of JPCAP. In Section V, we provide the tractability analysis for relaxations of JPCAP. Section VI proposes an algorithmic framework. Numerical results are given in Section VII. Conclusions are given in Section VIII.

\section{SySTEM MODEL}

\section{A. Basic Notation}

We consider a downlink cellular system with a base station (BS) serving $K$ users. The overall bandwidth $B$ is divided into $N$ subchannels, each with bandwidth $B / N$. Throughout the paper, we refer to subchannel interchangeably with subcarrier. We use $\mathcal{K}$ and $\mathcal{N}$ to denote the sets of users and subchannels, respectively, and $g_{k n}$ to denote the channel gain between the BS and user $k$ on subcarrier $n$. Let $p_{k n}$ be the power allocated to user $k$ on subcarrier $n$. A user $k$ is said to be multiplexed on a subchannel $n$, if and only if $p_{k n}>0$. The power values are 
subject to optimization. At the receiver, each user equipment has MUD capabilities to perform multi-user signal decoding. With SIC, some of the co-channel interference will be treated as decodable signals instead of as additive noise.

\section{B. NOMA Systems}

To ease the presentation of the system model, for the moment let us consider the case that all the $K$ users can multiplex on each subcarrier $n$ in a multi-carrier NOMA system (MC-NOMA) at downlink. For each subcarrier $n$, we sort the users in set $\mathcal{K}$ in the descending order of channel gains, and use bijection $b_{n}(k): \mathcal{K} \mapsto\{1,2, \ldots, K\}$ to represent this order, where $b_{n}(k)$ is the position of user $k \in \mathcal{K}$ in the sorted sequence. For our downlink system scenario, in [25] (Chapter 6.2.2, pp. 238) it is shown that, with superposition coding, a user can decode the data of another user with worse channel gain, and this is not constrained by the specific power split. The reason is that the first user, due to its better receiving condition than the second user, can decode any data that the second user can successfully decode. Consider one subcarrier and two users $k$ and $h$ with gain $g_{k}>g_{h}$. User $h$ does not perform SIC, and its rate equals $\log \left(1+\frac{p_{h} g_{h}}{p_{k} g_{h}+\eta}\right)$, where $\eta$ denotes the noise, and $p_{k}$ and $p_{h}$ are the power levels. For user $k$ with better gain $g_{k}$, the SINR for the data for user $h$ is $\frac{p_{h} g_{k}}{p_{k} g_{k}+\eta}>\frac{p_{h} g_{h}}{p_{k} g_{h}+\eta}$. Hence user $k$ can decode the data (at the rate governed by the right-hand side of the inequality) of user $h$, and this is not dependent on the power relation. Thus user $k$ is able to perform SIC, by subtracting the reencoded signal intended for receiver $h$ from the composite signal. That is, user $k$ on subcarrier $n$, before decoding its signal of interest, first decodes the received interfering signals intended for the users $h \in \mathcal{K} \backslash\{k\}$ that appear later in the sequence than $k$, i.e., $b_{n}(h)>b_{n}(k)$. The interfering signals with order $b_{n}(h)<b_{n}(k)$ will not be decoded and thus treated as noise. Hence, the interference after SIC for user $k$ on subcarrier $n$ is $\quad \sum \quad p_{h n} g_{k n}, \forall k \in \mathcal{K}, \forall n \in \mathcal{N}$. If $h \in \mathcal{K} \backslash\{k\}: b_{n}(h)<b_{n}(k)$

there are users having the same channel gain, then SIC applies following the principle in [25] (Chapter 6.2.2), provided that an ordering of the users is given. From the discussion, the SINR of user $k$ on subcarrier $n$ is given below.

$$
\mathrm{SINR}_{k n}=\frac{p_{k n} g_{k n}}{\sum_{h \in \mathcal{K} \backslash\{k\}: b_{n}(h)<b_{n}(k)} p_{h n} g_{k n}+\eta}
$$

The noise power here equals the product of the power spectral density of white Gaussian noise and the subcarrier bandwidth. The rate of each user in NOMA is determined by the user's SINR after SIC. Thus, the achievable rate of user $k$ on subcarrier $n$ is $R_{k n}=\log \left(1+\operatorname{SINR}_{k n}\right)$ nat/s with normalized bandwidth $\frac{B}{N}=1$.

For single-carrier NOMA systems (SC-NOMA), we omit the subcarrier index. For convenience, the users $k \in$ $\{1, \ldots, K\}$ in SC-NOMA are defined in the descending order of channel gains, where $g_{1} \geq g_{2} \geq, \ldots, \geq g_{K}$. Thus the user index also represents its position in the sequence, and user $k$ is able to decode the signal of user $h$ if $k<h$. We define $\operatorname{SINR}_{k}=\frac{p_{k} g_{k}}{\sum_{h \in \mathcal{K} \backslash\{k\}: h<k} p_{h} g_{k}+\eta}, \forall k \in \mathcal{K}$. The achievable rate of user $k$ is $R_{k}=\log \left(1+\operatorname{SINR}_{k}\right)$ nat $/ \mathrm{s}$ with normalized bandwidth $B=1$. Following the discussion earlier, for two users 1 and 2 with $g_{1}>g_{2}$, the achievable rates are $\log \left(1+\frac{p_{1} g_{1}}{\eta}\right)$ and $\log \left(1+\frac{p_{2} g_{2}}{p_{1} g_{2}+\eta}\right)$, respectively.

We use $\mathcal{U}_{n}$ as a generic notation for the set of users multiplexed on subchannel $n$ for MC-NOMA. For SC-NOMA, the corresponding entity is denoted by $\mathcal{U}$. We use $M, 1 \leq M \leq$ $K$, to denote the maximum number of multiplexed users on a subcarrier. The reason of having this parameter is to address complexity considerations of implementing MUD and SIC. In NOMA, the system complexity increases by $M$, because a user device needs to decode up to $M$ signals. The setting of $M$ depends on receiver's design complexity and signal processing delay for SIC [4], [8]. For practical implementation, $M$ is typically smaller than $K$. However, our optimization formulations and the solution algorithm are applicable to any value of $M$ between one and $K$. We also remark that the benefits of NOMA come with signaling overhead that is necessary to facilitate SIC. Although signaling is outside the scope of the current paper, it is of significance in practical implementation. On the other hand, it has been shown in [11] that NOMA remains superior to OMA in throughout when the signaling overhead is accounted for. In addition, parameter $M$, which limits the number of users per subcarrier, provides an effective way to control the signaling cost. By the results to be presented in Section VII, most of the improvements due to NOMA is achieved for small $M$.

Two utility functions, WSR and SR, are considered in this paper. The WSR utility is denoted by $f_{\mathrm{W}}=$ $\sum_{k \in \mathcal{K}} w_{k} \sum_{n \in \mathcal{N}} R_{k n}$, where $w_{k}$ is the weight coefficient of user $k \in \mathcal{K}$. Clearly, the selection of the weights has strong influence on the resource allocation among the users. In general, the weights can be used to steer the resource allocation towards various goals, such as to implement service class priority of users, and fairness (e.g., a user with averagely poor channel receives higher weight). In our work, the algorithmic approach is applicable without any assumption of the specific weight setting. For performance evaluation, we set the weights following proportional fairness. That is, for one time slot, a user's weight is set to be the reciprocal of the average user rate prior to the current time slot [15]. As a result, the resource allocation will approach proportional fairness over time. The SR utility, a special case of WSR, is defined as $f_{\mathrm{R}}=\sum_{k \in \mathcal{K}} \sum_{n \in \mathcal{N}} R_{k n}$. The term of SR utility is used interchangeably throughput in this paper. For both SR and WSR, SIC with superposition coding [25] applies to the users multiplexed on the same subcarrier. As was discussed earlier, the decoding does not rely on assuming specific, a priori constraint on the power allocation among the users.

\section{Joint Power And Channel Allocation}

In this section, we formulate JPCAP using WSR utility for MC-NOMA. We use W-JPCAP to denote the optimization problem. In general, JPCAP amounts to determining which users should be allocated to which subcarriers, as well as the optimal power allocation such that the total utility is maximized. In the following we define the variables and 
formulate $\mathrm{W}$-JPCAP as $P 1_{\mathrm{W} S R}$ below, where all $p$-variables and $x$-variables are collected in vectors $\boldsymbol{p}$ and $\boldsymbol{x}$, respectively.

$p_{k n}=$ allocated power to user $k$ on subcarrier $n$.

$x_{k n}= \begin{cases}1 & \text { if user } k \text { is multiplexed on subcarrier } n, \\ & \text { i.e., } p_{k n}>0, \\ 0 \quad \text { otherwise }\end{cases}$

$$
\begin{aligned}
P 1_{\mathrm{W} S R}: \max _{\boldsymbol{x}, \boldsymbol{p}} & \sum_{k \in \mathcal{K}} w_{k} \sum_{n \in \mathcal{N}} R_{k n} x_{k n} \\
\text { s.t. } & \sum_{k \in \mathcal{K}} \sum_{n \in \mathcal{N}} p_{k n} \leq P_{\text {tot }} \\
& \sum_{n \in \mathcal{N}} p_{k n} \leq P_{k}, \forall k \in \mathcal{K} \\
& \sum_{k \in \mathcal{K}} x_{k n} \leq M, \forall n \in \mathcal{N}
\end{aligned}
$$

In $P 1$, the objective ( $2 \mathrm{a}$ ) is to maximize the WSR utility, where $R_{k n}$ is defined below.

$$
R_{k n}=\log \left(1+\frac{p_{k n} g_{k n}}{\sum_{\substack{h \in \mathcal{K} \backslash\{k\}: \\ b_{n}(h)<b_{n}(k)}} p_{h n} g_{k n}+\eta}\right), \forall k \in \mathcal{K}, \forall n \in \mathcal{N}
$$

Constraints (2b) and (2c) are respectively imposed to ensure that the total power budget and the individual power limit for each user are not exceeded. The per-user power limit $P_{k}$ is introduced for practical considerations, such as regulatory requirement on power towards a user device. Such a limit is also very common in OMA (e.g., [23], [24]). Constraints (2d) restrict the maximum number of multiplexed users on each subcarrier to $M$. We remark that the power allocation is represented by the $p$-variables of which the values are subject to optimization, whereas the power limits $P_{t o t}$ and $P_{k}, k \in \mathcal{K}$ are given entities. Suppose $M$ users, say users $1, \ldots M$, are allocated with positive power on channel $n$. If $P_{t o t} \geq \sum_{k=1}^{M} P_{k}$ happens to hold, then all the $M$ users may be at their respective power limits, i.e., setting $p_{k n}=P_{k}, k=1, \ldots, M$, is feasible. Otherwise, the $M$ users can still be allocated with positive power, though not all of them can be at the power limits. Indeed, if user $k$ is allocated with power $p_{k n}>0$ on channel $n$, then typically $p_{k n}<P_{k}$ unless user $k$ is not allocated power on any other channel than $n$. For the total power limit $P_{\text {tot }}$ to be meaningful, one can assume $\sum_{k \in \mathcal{K}} P_{k}>P_{\text {tot }}$ without loss of generality, because otherwise the total power limit $P_{t o t}$ is not violated even if all users are allocated with their respective maximum power, that is, (2b) becomes void and should be dropped. We do not consider any further specific assumptions on the relation between $P_{\text {tot }}$ and $P_{k}, k \in \mathcal{K}$, to keep the generality of the system model.

Remark. We do not explicitly impose the constraint that $p_{k n}>0$ if and only if $x_{k n}=1$. This is because setting $p_{k n}>0$ and $x_{k n}=0$ is clearly not optimal, by the facts that $p_{k n}>0$ will lead to rate degradation of other users due to the co-channel interference (if there are other users on channel $n$ ), and that for user $k, p_{k n}>0$ means power is consumed, but $x_{k n}=0$ means no benefit as the rate in (2a) becomes zero. $\square$
Formulation $P 1_{\mathrm{W} S R}$ is non-linear and non-convex. The concavity of the objective function (2a) cannot be established in general, because of the presence of the binary $x$-variables and the product of $x$ and $p$. However, in complexity analysis, neither non-convexity nor non-linearity of a formulation proves the problem's hardness, as a problem could be inappropriately formulated. Therefore, we provide formal hardness analysis for W-JPCAP below.

\section{Theorem 1. W-JPCAP is NP-hard.}

Proof: We establish the result in two steps. First, we conclude that if $M=1$ in (2d), W-JPCAP is NP-hard, as it reduces to OFDMA subcarrier and power allocation, for which NP-hardness is provided in [22] and [23]. For general MC-NOMA with $M>1$, we construct an instance of $\mathrm{W}$ JPCAP and establish the equivalence between the instance and the OFDMA problem considered in [23]. We consider an instance of W-JPCAP with $K$ users, $N$ subcarriers, and $M=2$. Let $\epsilon$ denote a small value with $0<\epsilon<\frac{1}{e}^{K N}$. The total power $P_{t o t}$ is set to $N K P_{k}$. The power limit $P_{k}=1$ is uniform for $\forall k \in \mathcal{K}$, and the noise parameter $\eta=\epsilon$. Among the $K$ users, we select an arbitrary one, denoted by $\bar{k} \in \mathcal{K}$, and assign a dominating weight $w_{\bar{k}}=e^{K N}$ and channel gain $g_{\bar{k} n}=1$ on all the subcarriers, whereas the other users' weights and channel gains are $w_{k}=\epsilon$ and $g_{k n} \leq \frac{1}{e}^{K N}$, $\forall k \in \mathcal{K} \backslash\{\bar{k}\}$ and $\forall n \in \mathcal{N}$. From above, the ratios $\frac{w_{\bar{k}}}{w_{k}}$ and $\frac{g_{\bar{k} n}}{g_{k n}}$ are sufficiently large such that allocating any power $p \leq \stackrel{P_{\bar{k}}}{P}$ to user $\bar{k}$ on any subcarrier $n$, the utility $w_{\bar{k}} R_{\bar{k} n}>$ $\max \left(\sum_{k \in \mathcal{K} \backslash\{\bar{k}\}} \sum_{n \in \mathcal{N}} w_{k n} R_{k n}\right)$ for using the same power budget $p$, since $\sum_{k \in \mathcal{K} \backslash\{\bar{k}\}} \sum_{n \in \mathcal{N}} w_{k n} R_{k n}$ is bounded by $K N e^{-K N} \log \left(1+\frac{e^{-K N} p}{\epsilon}\right)$, and $w_{\bar{k}} R_{\bar{k} n}=e^{K N} \log \left(1+\frac{p}{\epsilon}\right)$ is clearly greater than $K N e^{-K N} \log \left(1+\frac{e^{-K N} p}{\epsilon}\right)$. Thus, allocating power to user $\bar{k}$ rather than other users is preferable for maximizing utility.

Due to the uniform gain $g_{\bar{k} n}$ and the dominating weight $w_{\bar{k}}$ for user $\bar{k}$ on all channels, the optimal power allocation for user $\bar{k}$ is to uniformly allocate an amount of $\frac{P_{\bar{k}}}{N}$ to each subcarrier. Then the remaining problem is to allocate power $P_{t o t}-P_{\bar{k}}=(N K-1) P_{k}$ to the remaining $K-1$ users. Every user $k \in \mathcal{K} \backslash\{\bar{k}\}$ is still subject to constraint (2c). Note that for $M=2$, each subcarrier now can accommodate one extra user at most. Compared to the OFDMA problem in [23], W-JPCAP has one extra total power constraint, i.e., (2b), however, recall that $P_{t o t}$ is set to $N K P_{k}$, and for this value (2b) is in fact redundant. Therefore, a special case of W-JPCAP with $M>1$ is equivalent to the OFDMA problem in [23], and the result follows.

\section{TRACTABILITY ANALYSIS FOR UNIFORM WEIGHTS}

The hardness of W-JPCAP could have stemmed from several sources, e.g., the structure of the utility function, discrete variables, non-concave objective, and the constraints. We start from investigating how the weight in the utility function influences the problem's tractability. The utility function can affect the computational complexity in problem solving [22], [24]. One example is that the SR maximization problem with total power constraint in OFDMA is polynomial-time 
solvable [22]. With WSR utility, solving the same problem is challenging [26]. In this section, we consider a special problem of W-JPCAP, i.e., SR utility with uniform weights for users. We use R-JPCAP to denote the optimization problem. Intuitively, R-JPCAP appears somewhat easier than W-JPCAP, however, the tractability of R-JPCAP, for both SC-NOMA and MC-NOMA, is not known in the literature. In the following, analogously to W-JPCAP, we formulate R-JPCAP in $P 1_{S R}$.

$$
P 1_{\mathrm{S} R}: \quad \max _{\boldsymbol{x}, \boldsymbol{p}} \sum_{k \in \mathcal{K}} \sum_{n \in \mathcal{N}} R_{k n} x_{k n}
$$

s.t. $(2 \mathrm{~b}),(2 \mathrm{c}),(2 \mathrm{~d})$

First, we provide structural insights for power allocation for R-JPCAP in SC-NOMA.

Theorem 2. For R-JPCAP in SC-NOMA, the following hold at the optimum:

(a) Suppose $g_{k} \geq g_{h}$ for two users $k$ and $h$ with $k \neq h$, then the optimal power allocation satisfies $p_{h}>0$ only if $p_{k}>0$.

(b) Up to $M$ consecutive users in descending order of gain are allocated with positive power.

Proof: By the SC-NOMA system model, the SR utility function $f=f\left(p_{1}, \ldots, p_{K}\right)_{\mathrm{R}}$ reads:

$\log \left(1+\frac{p_{1} g_{1}}{\eta}\right)+\log \left(1+\frac{p_{2} g_{2}}{p_{1} g_{2}+\eta}\right)+, \ldots,+\log \left(1+\frac{p_{K} g_{K}}{\sum_{h=1}^{K-1} p_{h} g_{K}+\eta}\right)$
$=\log \left(p_{1} g_{1}+\eta\right)-\log \eta+\log \left(\left(p_{1}+p_{2}\right) g_{2}+\eta\right)-$
$\log \left(p_{1} g_{2}+\eta\right)+, \ldots,+\log \left(\sum_{h=1}^{K} p_{h} g_{K}+\eta\right)-\log \left(\sum_{h=1}^{K-1} p_{h} g_{K}+\eta\right)$

Next, we consider the partial derivatives $\frac{\partial f}{\partial p_{1}}, \ldots$, $\frac{\partial f}{\partial p_{k}}, \ldots, \frac{\partial f}{\partial p_{K}}$, as shown in (5).

$$
\begin{aligned}
\frac{\partial f}{\partial p_{1}}= & \underbrace{\frac{g_{1}}{p_{1} g_{1}+\eta}-\frac{g_{2}}{p_{1} g_{2}+\eta}}_{\geq 0}+\underbrace{\frac{g_{2}}{\left(p_{1}+p_{2}\right) g_{2}+\eta}-\frac{g_{3}}{\left(p_{1}+p_{2}\right) g_{3}+\eta}}_{\geq 0}+ \\
, \ldots,+ & \underbrace{\frac{g_{K-1}^{K-1} p_{h} g_{K-1}+\eta}{\sum_{h=1}^{K-1} p_{h} g_{K}+\eta}}_{\geq 0}+\frac{g_{K}}{\sum_{h=1}^{K} p_{h} g_{K}+\eta},
\end{aligned}
$$

$\frac{\partial f}{\partial p_{2}}$

:

$$
\frac{\partial f}{\partial p_{K-1}}=\underbrace{\frac{g_{K-1}}{\sum_{h=1}^{K-1} p_{h} g_{K-1}+\eta}-\frac{g_{K}}{\sum_{h=1}^{K-1} p_{h} g_{K}+\eta}}_{\geq 0}+\frac{g_{K}}{\sum_{h=1}^{K} p_{h} g_{K}+\eta},
$$$$
\frac{\partial f}{\partial p_{K}}=\frac{g_{K}}{\sum_{h=1}^{K} p_{h} g_{K}+\eta}
$$

For conclusion (a), since $g_{1} \geq g_{2} \geq, \ldots, \geq g_{K}$ for the single subcarrier in SC-NOMA, it can be easily checked from
(5) that the partial derivatives $\frac{\partial f}{\partial p_{1}} \geq \frac{\partial f}{\partial p_{2}} \geq \frac{\partial f}{\partial p_{3}} \geq, \ldots, \geq$ $\frac{\partial f}{\partial p_{K}}>0$, irrespective of the power values. In general, from the partial derivatives, more utility will be obtained by increasing power $p_{k}$ instead of $p_{h}$ if $k<h, \forall k, h \in \mathcal{K}$. If the user with the best channel condition, i.e. user 1 , has $p_{1}<P_{1}$, the objective value $f\left(p_{1}, \ldots, p_{K}\right)_{R}$ can be improved by shifting power from other users to user 1 until $p_{1}$ equals the power limit $P_{1}$. Statement (a) also implies that the optimal power allocation will be in a consecutive manner, i.e., from user 1 to user $M$, one by one, and the result of (b) follows.

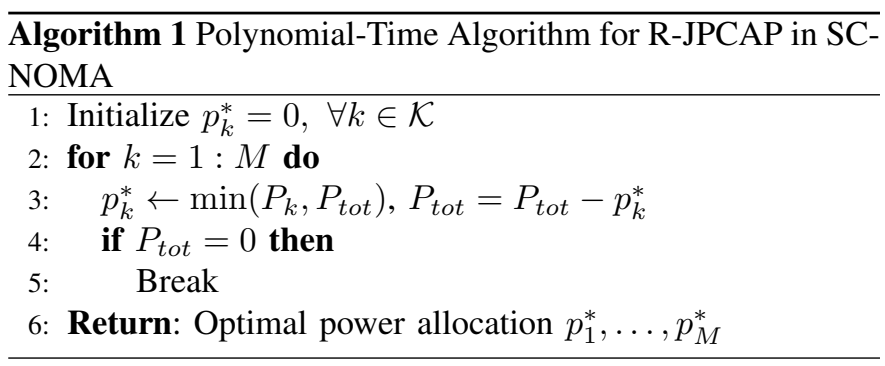

By Theorem 2, for the SR utility, the users being allocated positive power at optimum are consecutive in their gain values, starting from the user with the best channel gain. Note that the SR utility maximizes the throughput, but has the issue of fairness, which is addressed by the more general metric of weighted SR (WSR) utility, where the weights are set according to the proportional fairness policy. By Theorem 2 and its proof, R-JPCAP for SC-NOMA can be optimally solved by the procedure given in Algorithm 1. The users' power allocation is performed in a consecutive manner, starting from user $k=1$, and assigning power $\min \left(P_{k}, P_{t o t}\right)$ to user $k$ and updating $P_{\text {tot }}$ accordingly. Algorithm 1 is clearly of polynomial-time complexity, resolving the tractability of R-JPCAP in SC-NOMA, giving Corollary 3 below.

Corollary 3. R-JPCAP for SC-NOMA is tractable, i.e., polynomial-time solvable.

Next, we analyze the computational complexity of R-JPCAP in MC-NOMA.

\section{Theorem 4. R-JPCAP for MC-NOMA is NP-hard.}

Proof: The proof is analogous to that of Theorem 1. For general R-JPCAP with $M>1$, we construct a special instance with $K$ users, $N$ subcarriers, $M=2, P_{t o t}=N K P_{k}$, and uniform $P_{k}=1$. We deploy a "dominant user" $\bar{k} \in \mathcal{K}$ in the instance, that is, user $\bar{k}$ has the highest and uniform channel gain of $g_{\bar{k} n}=1$ for all the subcarriers, whereas the channel gain of all the other users and subcarriers is $g_{k n} \leq \frac{1}{e}^{K N}$, $\forall k \in \mathcal{K} \backslash\{\bar{k}\}, \forall n \in \mathcal{N}$. One can observe that the ratio $\frac{g_{\bar{k} n}}{g_{k n}}$ for $\forall n \in \mathcal{N}, \forall k \in \mathcal{K} \backslash\{\bar{k}\}$ has been set sufficiently large, and from (5), the partial derivative of user $\bar{k}$ satisfies $\frac{\partial f}{\partial p_{\bar{k} n}}>\frac{\partial f}{\partial p_{k n}}$ for $\forall k \in \mathcal{K} \backslash\{\bar{k}\}$ on each $n$, irrespective of the power values. Then the statement (a) in Theorem 2 is valid for any subcarrier $n$ in this instance, that is, if $\bar{k}$ is multiplexed on subcarrier $n$, the optimal power $p_{k n}>0$ only if $p_{\bar{k} n}>0$ for any $k \neq \bar{k}$. Thus, on each subcarrier $n$, allocating power to user $\bar{k}$ is preferred for optimality. Furthermore, due to the uniform 
channel gain for user $\bar{k}$, the optimal power allocation for user $\bar{k}$ is to allocate equal power $\frac{1}{N}$ on every subcarrier. Then the remaining problem is equivalent to the OFDMA resource allocation problem in [23], and the result follows.

From the results in this section, using SR utility function instead of WSR does not change the intractability of JPCAP.

\section{TRACTABILITY ANALYSIS FOR RELAXED JPCAP}

In this section, we aim to identify and characterize tractable cases for JPCAP. We provide tractability and convexity analysis for a relaxed version of W-JPCAP and R-JPCAP. For problem's relaxation, we make the following observations. First, from the proofs of Theorem 1 and 4, we have the following corollary.

Corollary 5. Both R-JPCAP and W-JPCAP remain NP-hard, even if constraint $(2 b)$ is relaxed (i.e., the constraint is removed from the optimization formulations $P 1_{S R}$ and $\left.P 1_{W S R}\right)$.

Second, solving JPCAP will be challenging if (2c) is present. The observation applies also to OFDMA resource allocation, see e.g., [1], [22], [23]. Next, the discrete $x$ variables are introduced in JPCAP due to the presence of constraint (2d). This results in a non-convex feasible region. In the following, we relax two constraints (2c) and (2d) as well as removing $x$-variables, and construct a relaxed version of R-JPCAP and W-JPCAP in $P 2_{\mathrm{S} R}$ and $P 2_{\mathrm{W} S R}$, respectively.

$$
\begin{aligned}
P 2_{\mathrm{S} R}: & \max _{p} \sum_{k \in \mathcal{K}} \sum_{n \in \mathcal{N}} R_{k n}, \quad \text { s.t. } \\
P 2_{\mathrm{W} S R}: & \max _{p} \sum_{k \in \mathcal{K}} w_{k} \sum_{n \in \mathcal{N}} R_{k n}, \quad \text { s.t. }
\end{aligned}
$$

Note that both formulations above are with the $p$-variables only. We characterize the optimal power allocation for $P 2_{\mathrm{SR}}$ in SC-NOMA first.

Lemma 6. For $P 2_{S R}$ in $S C$-NOMA with $g_{1} \geq g_{2} \geq, \ldots, \geq g_{K}$, power allocation $p_{1}=P_{t o t}, p_{2}=\cdots=p_{K}=0$ is optimal.

Proof: First, observe that relaxing the user-individual power constraint (2c) is equivalent to setting $P_{k}=P_{t o t}$, i.e., the user power limit is set to be equal to the total power limit, such that (2c) becomes redundant. Then, the result of the lemma is obtained by applying the result of Theorem 2, that is, the optimum can be computed by using Algorithm 1, which, with $P_{k}=P_{t o t}$, leads immediately to the result of the lemma.

Next, we generalize the results of Lemma 6 to multi-carrier systems, and show $P 2_{\mathrm{SR}}$ is also tractable in MC-NOMA.

Lemma 7. For $P 2_{S R}$ in MC-NOMA, there is an optimal solution satisfying $\left|\mathcal{U}_{n}\right| \leq 1, \forall n \in \mathcal{N}$, i.e., OMA is optimal.

Proof: Suppose at the global optimum, a subcarrier $n$ has $\left|\mathcal{U}_{n}\right|>1$. Consider the two users having the largest gain values in $\mathcal{U}_{n}$, and, without any loss of generality of the proof, suppose that the two user indices are 1 and 2, with $g_{1 n} \geq g_{2 n}$. Denote the power allocated to users 1 and 2 on this subcarrier by $p_{1 n}$ and $p_{2 n}$, respectively, with $p_{1 n}>0$ and $p_{2 n}>0$. Denote by $p_{n}^{*}$ the sum of the two, i.e., $p_{n}^{*}=p_{1 n}+p_{2 n}$. The achieved sum utility for these two users is thus $R_{n}^{(2)}=\log \frac{p_{1 n} g_{1 n}+\eta}{\eta}+$ $\log \frac{p_{n}^{*} g_{2 n}+\eta}{p_{1 n} g_{2 n}+\eta}$.

Consider allocating the amount of power $p_{n}^{*}$ to user 1 instead, leaving zero power to user 2 . The power allocation of the other users remain unchanged. Note that this power re-allocation affects only the utility values of user 1 and 2 . For user 1 the resulting utility becomes $R_{n}^{(1)}=\log \frac{p_{n}^{*} g_{1 n}+\eta}{\eta}$, whereas for user 2 the utility is zero. Comparing $R_{n}^{(1)}$ and $R_{n}^{(2)}$, we obtain the following.

$$
\begin{aligned}
& R_{n}^{(2)}-R_{n}^{(1)}=\log \left(\frac{p_{1 n} g_{1 n}+\eta}{p_{n}^{*} g_{1 n}+\eta} \times \frac{p_{n}^{*} g_{2 n}+\eta}{p_{1 n} g_{2 n}+\eta}\right) \\
& =\log \left(\frac{p_{1 n} g_{1 n} p_{n}^{*} g_{2 n}+p_{1 n} g_{1 n} \eta+p_{n}^{*} g_{2 n} \eta+\eta^{2}}{p_{1 n} g_{1 n} p_{n}^{*} g_{2 n}+p_{n}^{*} g_{1 n} \eta+p_{1 n} g_{2 n} \eta+\eta^{2}}\right)
\end{aligned}
$$

It can be observed that

$$
\begin{aligned}
& \left(p_{1 n} g_{1 n} \eta+p_{n}^{*} g_{2 n} \eta\right)-\left(p_{n}^{*} g_{1 n} \eta+p_{1 n} g_{2 n} \eta\right) \\
& =\left(p_{1 n} \eta-p_{n}^{*} \eta\right)\left(g_{1 n}-g_{2 n}\right)<0
\end{aligned}
$$

Since $p_{1 n}<p_{n}^{*}$ and $g_{1 n}>g_{2 n}$, we have $R_{n}^{(2)}<R_{n}^{(1)}$. This contradicts the optimality of the first power allocation, and the lemma follows.

From Lemma 7 for $P 2_{\mathrm{SR}}$, which amounts to maximizing the sum rate utility subject to one single constraint on the total power, OMA resource allocation is optimal. We remark that $P 2_{\mathrm{SR}}$ is convex and tractable. It can be checked that the Hessian matrix of the objective function in $P 2_{\mathrm{SR}}$ is negative semi-definite. As the $p$-variables are continuous and $(2 \mathrm{~b})$ is linear, $P 2_{\mathrm{SR}}$ is convex. In general, the optimal solution can be obtained by performing a polynomial-time algorithm [22], that is, choosing the user with the best channel gain on each subcarrier and then applying water-filling power allocation for the assigned users. The conclusion is summarized below.

Corollary 8. The optimization problem in $P 2_{S R}$ is tractable for SC-NOMA and MC-NOMA.

Remark. In some proposed NOMA schemes, e.g., [9], [10], users with inferior channel condition may request more power to enhance fairness, e.g., if $g_{1 n} \geq g_{2 n} \geq, \ldots, \geq g_{K n}$ on a subcarrier $n$, the power allocation is subject to $0<p_{1 n} \leq$ $p_{2 n} \leq, \ldots, \leq p_{K n}$. By Lemma 6, we remark that, on each subcarrier $n$, equal power allocation for the multiplexed users $k \in \mathcal{U}_{n}$ is optimal.

In the following, we characterize the tractability and convexity for $P 2_{\mathrm{WSR}}$. From formulation $P 2_{\mathrm{WSR}}$, the convexity is not straightforward to obtain. Note that Lemma 6 and Lemma 7 may not hold for $P 2_{\mathrm{WSR}}$, as a user with inferior channel gain may be associated with higher weight, and as a result, the optimum possibly has $\left|\mathcal{U}_{n}\right|>1$ for some $n \in \mathcal{N}$. We make the following derivations to show $P 2_{\mathrm{WSR}}$ is convex.

Theorem 9. The optimization problem in $P 2_{W S R}$ is convex.

Proof: From Eq. (3), for any subcarrier $n \in \mathcal{N}$, there are $K$ equations linking the power allocation with the user rates. For the user with the best gain value, there is no interference term in Eq. (3), and hence the power variable of this user can be expressed in its rate. Going through the remaining users in descending order of gain and performing successive variable 
substitution, the $p$-variables can be all expressed in the rate values. Utilizing the observation, we prove the convexity by reformulating $P 2_{\mathrm{WSR}}$ by treating rates $R_{k n}, k \in \mathcal{K}, n \in \mathcal{N}$ as the optimization variables. This transformation is analogous to the geometric programming method [27]. To facilitate the proof, we use $m_{n}(i)$ to denote the user index in the $i$ th position in the sorted sequence for subcarrier $n$, with indices $i=0, \ldots, K$, and the convention that $m_{n}(0)=0$. Problem $P 2_{\mathrm{WSR}}$ is then reformulated below.

$$
\begin{aligned}
& \max _{\boldsymbol{R}} \sum_{i=1}^{K} w_{k} \sum_{n=1}^{N} R_{k n} \\
& \text { s.t. } \sum_{i=1}^{K} \sum_{n=1}^{N}\left(\frac{\eta}{g_{m_{n}(i), n}}-\frac{\eta}{g_{m_{n}(i-1), n}}\right) \exp \left(\sum_{h=i}^{K} R_{m_{n}(h), n}\right) \\
& \quad-\frac{\eta}{g_{m_{n}(K), n}} \leq P_{t o t} \\
& \quad R_{k n} \geq 0, \forall k \in \mathcal{K}, \forall n \in \mathcal{N}
\end{aligned}
$$

The objective and constraints (10c) are both linear. For constraints (10b), note that $\frac{\eta}{g_{m_{n}(i), n}}-\frac{\eta}{g_{m_{n}(i-1), n}} \geq 0$, due to the descending order of channel gains, Hence the sum-exp function in (10b) is convex [28], and the theorem follows.

For the above convex and tractable cases, i.e., $P 2_{\mathrm{SR}}$ and $P 2_{\mathrm{WSR}}$, standard optimization approaches for convex problem can be applied. For intractable cases, we develop an algorithmic framework based on Lagrangian dual optimization and dynamic programming (DP) to provide both near-optimal solutions and optimality bounds in the next section.

\section{OPTIMIZATION AlgORITHM FOR NOMA POWER AND CHANNEL ALLOCATION}

In view of the complexity results, we aim to develop an algorithm that is not for exact global optimum, yet the algorithm by design, is capable of providing near-optimal solutions. Moreover, the algorithm is expected to deliver optimality bounds in order to gauge performance, and is capable of progressively improving the bounds by scaling parameters. In this section, we propose an algorithmic framework based on Lagrangian duality and dynamic programming (LDDP). In the developed algorithm, we make use of the Lagrangian dual from relaxing the individual power constraint (2c) with multipliers, and we develop a DP based approach to solve the problem for given multipliers. The algorithm is designed to solve both R-JPCAP and W-JPCAP problems. For generality, we take W-JPCAP for illustration.

\section{A. Lagrangian Duality and Power Discretization}

Let vectors $\boldsymbol{p}$ and $\boldsymbol{x}$ collect all $p$-variables and $x$-variables, respectively. Vector $\boldsymbol{\lambda}:=\left\{\lambda_{k}, \forall k \in \mathcal{K}\right\}$ contains the Lagrangian multipliers associated with constraints (2c) in $P 1_{\mathrm{WSR}}$. We construct the subproblem of Lagrangian relaxation below.

$P_{\mathrm{LR}}: \max _{\boldsymbol{x}, \boldsymbol{p}} L(\boldsymbol{x}, \boldsymbol{p}, \boldsymbol{\lambda})=\sum_{k \in \mathcal{K}} w_{k} \sum_{n \in \mathcal{N}} x_{k n} R_{k n}+\sum_{k \in \mathcal{K}} \lambda_{k}\left(P_{k}-\sum_{n \in \mathcal{N}} p_{k n}\right)$

s.t. $(2 \mathrm{~b}),(2 \mathrm{~d})$

$P_{\mathrm{LR}}$ is subject to the total power constraint (2b) as well as constraints $(2 \mathrm{~d})$ that limit the number of users in each subcarrier. Unlike the objective in $P 1_{\mathrm{WSR}}$ and $P 1_{\mathrm{SR}}$, allocating power to user $k$ on subcarrier $n$ in $P_{\mathrm{LR}}$ requires to pay a penalty in utility, i.e., $-\lambda_{k} p_{k n}$. The Lagrange dual function is defined by $z(\boldsymbol{\lambda})=\max _{\boldsymbol{x}, \boldsymbol{p}} L(\boldsymbol{x}, \boldsymbol{p}, \boldsymbol{\lambda})$. The dual optimum is correspondingly defined below.

$$
z^{*}=\min _{\boldsymbol{\lambda} \succeq 0} z(\boldsymbol{\lambda})
$$

The optimization task amounts to solving $P_{\mathrm{LR}}$ for a given $\boldsymbol{\lambda}$ and finding the optimal $\boldsymbol{\lambda}$ to minimize the Lagrangian dual in (12). Note that since $P 1_{\mathrm{W} S R}$ and $P 1_{\mathrm{S} R}$ are non-convex in general, there may exist a duality gap between $z^{*}$ and global optimum $z^{\dagger}$ to the original problem, i.e., $z^{*} \geq z^{\dagger}$.

Formulation $P_{\mathrm{LR}}$ is non-convex in general due to the reasons we discussed in Section IV. We consider solving $z(\boldsymbol{\lambda})$, making use of the observation that, once the power is discretized, $P_{\mathrm{LR}}$ admits the use of DP for reaching optimality in polynomialtime of the problem size and the number of power discretization levels. To this end, we discretize the power budget $P_{t o t}$ into $J$ uniform steps, and denote by $\delta$ the size of each step, i.e., $\delta=P_{\text {tot }} / J$. Denote by $p^{j}$ be the power value for level $j$ and $p^{j}=\delta * j$, where $j \in \mathcal{J}=\{1, \ldots, J\}$. We denote by $P_{\text {LR-D }}$ the version of $P_{\mathrm{LR}}$ after power discretization. The formulation of $P_{\text {LR-D }}$ and its optimization variables are presented below.

$$
\begin{aligned}
& x_{k n}^{j}=\left\{\begin{array}{l}
1 \quad \begin{array}{l}
\text { if power level } j \text { is allocated to user } k \text { on } \\
\text { subcarrier } n, \\
0 \quad \text { otherwise. }
\end{array}
\end{array}\right. \\
& P_{\text {LR-D }}: \quad \max _{\boldsymbol{x}} L_{D}(\boldsymbol{x}, \boldsymbol{\lambda})=\sum_{k \in \mathcal{K}} w_{k} \sum_{n \in \mathcal{N}} \sum_{j \in \mathcal{J}} x_{k n}^{j} R_{k n}^{j}+ \\
& \sum_{k \in \mathcal{K}} \lambda_{k}\left(P_{k}-\sum_{n \in \mathcal{N}} \sum_{j \in \mathcal{J}} x_{k n}^{j} p^{j}\right) \\
& \text { s.t. } \quad \sum_{k \in \mathcal{K}} \sum_{n \in \mathcal{N}} \sum_{j \in \mathcal{J}} x_{k n}^{j} p^{j} \leq P_{\text {tot }} \\
& \sum_{k \in \mathcal{K}} \sum_{j \in \mathcal{J}} x_{k n}^{j} \leq M, \forall n \in \mathcal{N} \\
& \sum_{j \in \mathcal{J}} x_{k n}^{j} \leq 1, \forall k \in \mathcal{K}, \forall n \in \mathcal{N}
\end{aligned}
$$

The objective (13a) and constraints (13b), (13c) originate from $P_{\mathrm{LR}}$ but are adapted to power discretization. In (13a), the achievable rate of allocating user $k$ on subcarrier $n$ with power level $j$ is denoted by $R_{k n}^{j}$ below for $\forall k \in \mathcal{K}, \forall n \in \mathcal{N}$, $\forall j \in \mathcal{J}$, and with normalized bandwidth $\frac{B}{N}=1$.

$$
R_{k n}^{j}=\log \left(1+\frac{p^{j} g_{k n}}{\sum_{h \in \mathcal{K} \backslash\{k\}: b_{n}(h)<b_{n}(k)}\left(\sum_{j^{\prime} \in \mathcal{J}} x_{h n}^{j^{\prime}} p^{j^{\prime}}\right) g_{k n}+\eta}\right)
$$

Constraints (13d) state that each user on a subcarrier can select one power level at most, and $\sum_{j \in \mathcal{J}} x_{k n}^{j}=0$ means that there is no power allocation for user $k$ on subcarrier $n$. For given $\boldsymbol{\lambda}$, let $z_{D}(\boldsymbol{\lambda})=\max L_{D}(\boldsymbol{x}, \boldsymbol{\lambda})$ and $\boldsymbol{p}^{*}$ denote the optimal objective value and the corresponding power solution of $P_{\text {LR-D }}$, respectively. Next, we develop a DP based approach to solve $P_{\text {LR-D }}$ exactly to optimality.

Remark. Power discretization in $P_{\text {LR-D }}$ is considered as an approximation for the continuous power allocation in $P_{\mathrm{LR}}$. 
However, in practical systems, the power is typically set in discrete steps, e.g., discrete power control in LTE downlink [29]. In this case the discrete model $P_{\text {LR-D }}$ is exact.

\section{B. Two-Stage DP Based Approach}

Given power levels in set $\mathcal{J}$ and multipliers in vector $\lambda$, problem $P_{\text {LR-D }}$ can be solved by using DP. In general, DP guarantees global optimality if the problem has the so called "optimal substructure property" [30]. A classical example is the knapsack problem with integer coefficients [31]. In our case, $P_{\text {LR-D }}$ does exhibit the property, and a proof of the optimality of DP will be provided later in Theorem 10.

To ease the presentation, we describe the DP algorithm in two stages. In the first stage, intra-subcarrier power allocation is carried out among users, that is, for subcarrier $n \in \mathcal{N}$, the algorithm computes the optimal user power allocation by treating power $p^{j}, j=1, \ldots, J$, as the power budget for the subcarrier in question. The optimal utility value of consuming power $p^{j}$ on $n$ is denoted by $V_{n, j}$, where $n \in \mathcal{N}, j \in \mathcal{J}$. Since the number of multiplexed users cannot exceed $M$ on each subcarrier, we keep track on the optimum allocation for each $m \in\{1, \ldots, M\}$. We define a tuple of format $t=\left(u_{t}, M_{t}\right)$ to represent a candidate partial solution, where $u_{t}$ is the utility value, and $M_{t}$ is the number of users allocated with positive power. For a partial problem of assigning positive power to exactly $m$ out of the first $k$ users with power budget $p^{j}$, the optimal utility is denoted by $T_{k, j}^{m}$.

The optimality of stage 1 is obtained from DP recursion. The values $T_{k, j}^{m}$ can be arranged in form of a $K \times J \times M$ matrix $\mathcal{A}_{1}$. Computing $T_{k, j}^{m}$ for $k, m, j=1$ is straightforward. For $k \geq 2, m \geq 2$ and $j \geq 2$, the following recursive formula is used to obtain the corresponding value in $\mathcal{A}_{1}$.

$$
T_{k, j}^{m}=\max \left\{\max _{j^{\prime}=1, \ldots, j-1}\left\{w_{k} R_{k n}^{j^{\prime}}-\lambda_{k} p^{j^{\prime}}+T_{k-1, j-j^{\prime}}^{m-1}\right\}, T_{k-1, j}^{m}\right\}
$$

From (14), the procedure of obtaining $T_{k, j}^{m}$ is decomposed into multiple stages, and the recursion is applied to move from one stage to another. Thus, each partial problem has an optimal substructure [30].

The algorithmic operations for stage one are given in Algorithm 2. The bulk of the computation starts at Line 2 . For user $k=1$, exactly one tuple is created for each $j$, and the corresponding utility value is $T_{1, j}^{1}, j \in \mathcal{J}$, see Line 4 . For users $k>1$, the recursion is performed in Lines 5 to 15 . In Lines 9 to 15 , a tuple $t$ is created, and its utility value $u_{t}$ will replace the current $T_{k, j}^{m}$ if $u_{t}>T_{k, j}^{m}$. Note that in Line 12 , the utility $u_{t}$ is the sum of two parts, i.e., assigning a trial power $p^{j^{\prime}}$ to user $k$ plus the previously obtained maximum utility $T_{k-1, j-j^{\prime}}^{m-1}$. The algorithm terminates when all the $K$ users and $J$ power levels have been processed. The optimal value of assigning power $p^{j}$ on a subcarrier $n$ is stored in $V_{n, j}$, see Line 17.

In the second stage, power allocation of $P_{t o t}$ is carried out among the subcarriers, i.e., inter-subcarrier power allocation. Then DP is applied to perform optimal power allocation at the subcarrier level. For given $\boldsymbol{\lambda}, z_{D}(\boldsymbol{\lambda})$ is obtained in Algorithm 3. The operations start at Line 3. In the first stage of TSDP, Algorithm 2 is performed to obtain $V_{n, 1}, \ldots, V_{n, J}$ for each

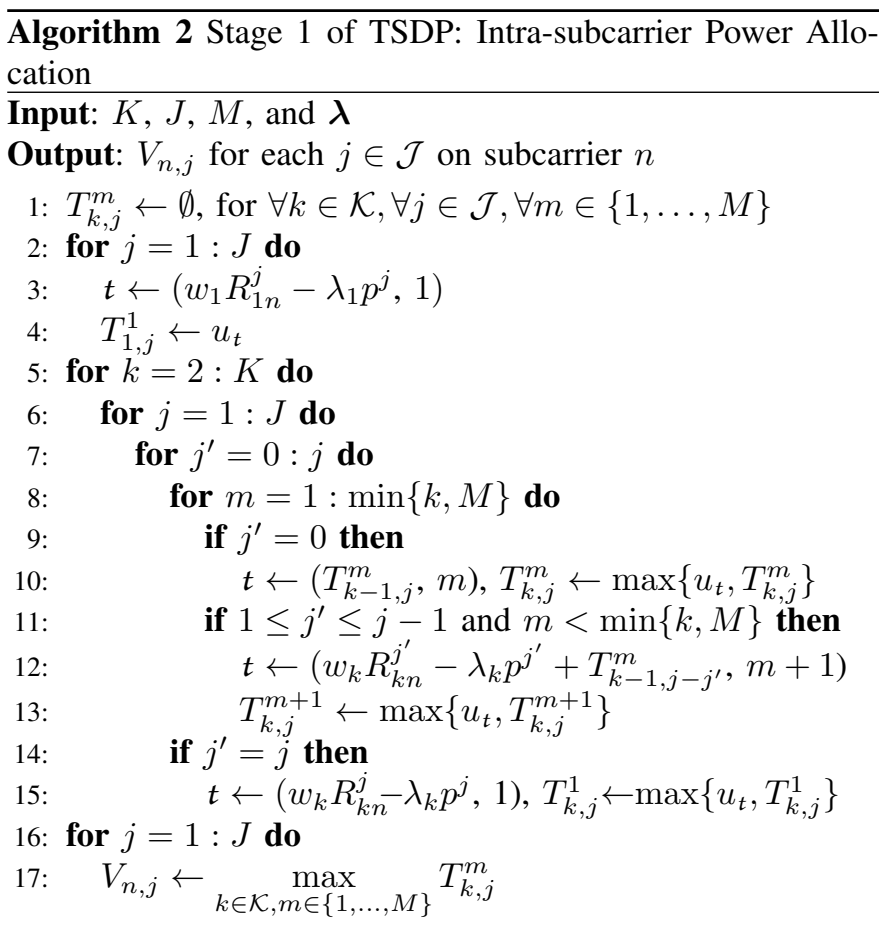

subcarrier $n \in \mathcal{N}$. Note that by the construction of Algorithm 2 , in $V_{n, j}$, the number of multiplexed users is at most $M$ for $\forall n \in \mathcal{N}$. Thus in stage two, index $m$ is no longer needed. In Lines 6-10, based on the accumulated value $\hat{T}_{n-1, j-j^{\prime}}$, a new candidate for $\hat{T}_{n, j}$ is obtained by adding $V_{n, j^{\prime}}$. Then for the partial problem of allocating power $p^{j}$ to $1, \ldots, n$ subcarriers, the optimal solution $\hat{T}_{n, j}$ is obtained.

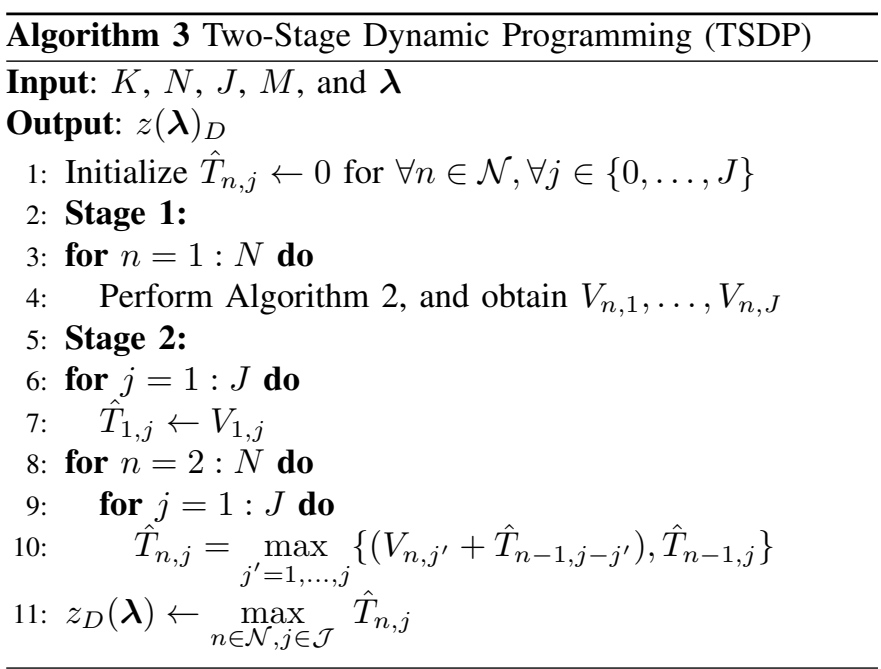

The DP recursion (for $n \geq 2$ ) for the second stage is given in Line 10. The values $\hat{T}_{n, j}$ for all $k \in \mathcal{K}, j \in \mathcal{J}$ can be viewed in form of an $N \times J$ matrix $\mathcal{A}_{2}$. From the DP recursions in TSDP, the global optimum of $P_{\mathrm{LR}-\mathrm{D}}$ is obtained from accumulating the solutions of the partial problems. By the end of stage two, $z_{D}(\boldsymbol{\lambda})$ is equal to the maximum $\hat{T}_{n, j}$ among the elements in $\mathcal{A}_{2}$, see Line 11 . In $P_{\mathrm{LR}-\mathrm{D}}$, for the partial problem for users $1, \ldots, k$, subcarriers $1, \ldots, n$, and with a total power budget $p^{j}$, the optimum is independent of 
that for the remaining subcarriers or users. The complexity for computing optimality is provided in Theorem 10.

Theorem 10. The global optimum of $P_{L R-D}$ is obtained by TSDP with a time complexity being polynomial in $M, N, K$, and $J$.

Proof: The input of $P_{\text {LR-D }}$ are $N, K, M$ and $J$. By inspecting (14) and Line 10 in Algorithm 3, computing matrix $\mathcal{A}_{1}$ for all subcarriers requires $\mathcal{O}\left(K N M J^{2}\right)$ in running time. For matrix $\mathcal{A}_{2}$, the running time is of $\mathcal{O}\left(N J^{2}\right)$. Hence the former is dominating, and the overall time complexity is $\mathcal{O}\left(K N M J^{2}\right)$, which is polynomial in the input size.

Remark. Increasing $J$ provides better granularity in power discretization. By improving the granularity, the solution of $P_{\text {LR-D }}$ can approach arbitrarily close to that of $P_{\mathrm{LR}}$.

\section{Algorithmic Framework: Lagrangian Duality With Dy- namic Programming}

We develop a framework LDDP to deliver near-optimal solutions (N-LDDP) of the global optimum $z^{\dagger}$ of $P 1_{\mathrm{W} S R}$ and $P 1_{\mathrm{S} R}$. We also derive a scheme UB-LDDP to provide upper bounds for gauging the solution quality of N-LDDP. LDDP is summarized in Algorithm 4. In Line 3 to Line 11, we obtain $z_{D}(\boldsymbol{\lambda})$ and the power solution $\boldsymbol{p}^{*}$ by applying TSDP to solve $P_{\text {LR-D }}$. These steps constitute N-LDDP. The iterations for solving the Lagrangian dual terminate either after a specified number of iterations $C_{\max }$, or if the difference between the objective values in two successive iterations is less than $\epsilon$ [30]. Lines 15-20 form UB-LDDP, which approximates the global optimum from above. UB-LDDP delivers an upper bound, i.e., a value that is guaranteed to be no smaller than the global optimum. The purpose is for performance evaluation. Note that the problem is maximization, and hence the solution from the first part of the algorithm, N-LDDP, has a utility value that is lower than global optimum. Computing the global optimum is NP-hard. However, to assess the performance of this solution, we can instead obtain the upper bound at significantly lower complexity. The deviation from global optimum cannot be more than the deviation from the upper bound, which can be used in our performance evaluation.

In Line 9, the users' individual power constraints may be violated in $\boldsymbol{p}^{*}$. Thus, we develop a three-step approach to convert $\boldsymbol{p}^{*}$ into a feasible solution $\boldsymbol{p}_{f}$ for JPCAP if the former violates (2c). Let set $\overline{\mathcal{K}}$ denote the users allocated with positive power in $\boldsymbol{p}^{*}, \overline{\mathcal{K}} \subseteq \mathcal{K}$. We denote $\mathcal{K}^{\prime}=\left\{k \in \overline{\mathcal{K}}: \sum_{n \in \mathcal{N}} p_{k n}>P_{k}\right\}$ as the subset of users for which (2c) does not hold. To obtain $\boldsymbol{p}_{f}$, step one, power allocation for each user $k \in \overline{\mathcal{K}} \backslash \mathcal{K}^{\prime}$ on subcarriers keeps same as in $\boldsymbol{p}^{*}$. Step two, for each $k \in \mathcal{K}^{\prime}$, the subcarriers are sorted in ascending order in power allocation of $k$. Following the sequence, power is allocated as in $\boldsymbol{p}^{*}$, however until the limit $P_{k}$ is reached. Doing so releases an amount of $\sum_{k \in \mathcal{K}^{\prime}} \sum_{n \in \mathcal{N}} p_{k n}-\sum_{k \in \mathcal{K}^{\prime}} P_{k}$ power that can be re-allocated to users in $\mathcal{K} \backslash \mathcal{K}^{\prime}$ in step three. The re-allocation follows the descending order of the product of channel gain and weight. That is, letting $(\tilde{k}, \tilde{n})=\operatorname{argmax} w_{k n} g_{k n}, k \in$ $\overline{\mathcal{K}} \backslash \mathcal{K}^{\prime}, n \in \mathcal{N}, p_{\tilde{k} \tilde{n}}$ is increased as much as allowed by $P_{\tilde{k}}$ and $P_{\text {tot }}$, then we select the next best candidate, and so on,

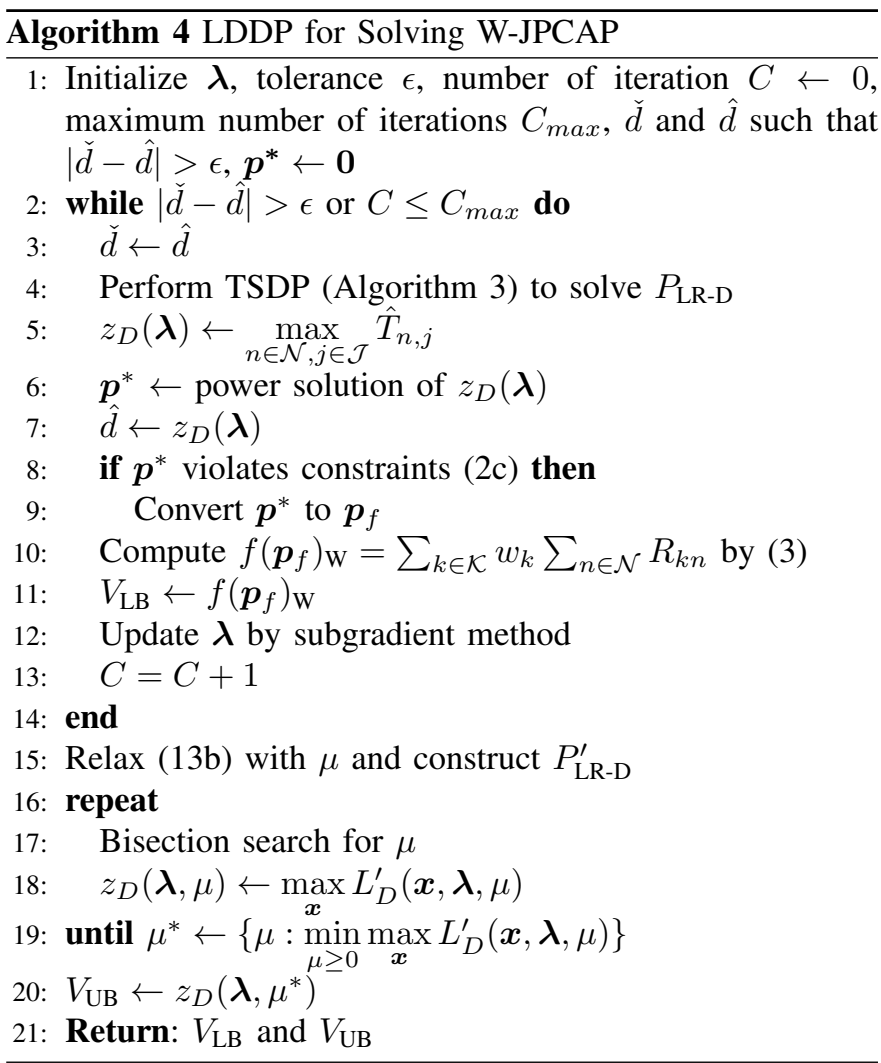

until either $P_{\text {tot }}$ or $P_{k}$ for all $k \in \overline{\mathcal{K}}$ is reached. In Line 10, we obtain the resulting utility $f\left(\boldsymbol{p}_{f}\right)_{\mathrm{W}}$ for using $\boldsymbol{p}_{f}$, where the calculation of $f\left(\boldsymbol{p}_{f}\right)_{\mathrm{W}}=\sum_{k \in \mathcal{K}} w_{k} \sum_{n \in \mathcal{N}} R_{k n}$ follows the equation in (3). The utility value of N-LDDP is delivered in $V_{\mathrm{LB}}$ at Line 11 . In Line $12, V_{\mathrm{LB}}$ is used in the calculation of step size of subgradient optimization (see [30]).

To provide an upper bound for $z^{\dagger}$, we apply post-processing to $z_{D}(\boldsymbol{\lambda})$ in LDDP. We remark that if $J$ is sufficiently large, $z_{D}(\boldsymbol{\lambda})$ can be empirically considered as an upper bound to W-JPCAP or R-JPCAP due to Lagrangian duality, however, theoretically there is no guarantee. For example, $z_{D}(\boldsymbol{\lambda})$ could be possibly less than $z^{\dagger}$ for small $J$, e.g., $J=1$. From Line 15 to Line 20, we design an approach to convert $z_{D}(\boldsymbol{\lambda})$ to a theoretically guaranteed upper bound. First, in Line 15, for the multipliers in $\boldsymbol{\lambda}$ and keeping their values fixed, we further relax the total power constraint (13b) with multiplier $\mu$, and reconstruct the subproblem of Lagrangian relaxation below.

$$
\begin{gathered}
P_{\text {LR-D }}^{\prime}: \max _{\boldsymbol{x}} L_{D}^{\prime}(\boldsymbol{x}, \boldsymbol{\lambda}, \mu)=\underbrace{\sum_{k \in \mathcal{K}} w_{k} \sum_{n \in \mathcal{N}} \sum_{j \in \mathcal{J}} x_{k n}^{j} \bar{R}_{k n}^{j}}_{\text {part I }}+ \\
\underbrace{\sum_{k \in \mathcal{K}} \lambda_{k}\left(P_{k}-\sum_{n \in \mathcal{N}} \sum_{j \in \mathcal{J}} x_{k n}^{j}\left(p^{j}-\delta\right)\right)}_{\text {part II }}+\underbrace{\mu\left(P_{t o t}-\sum_{k \in \mathcal{K}} \sum_{n \in \mathcal{N}} \sum_{j \in \mathcal{J}} x_{k n}^{j}\left(p^{j}-\delta\right)\right)}_{\text {part III }} \\
\text { s.t. (13c) and (13d) }
\end{gathered}
$$

The calculation of $\bar{R}_{k n}^{j}$ for $\forall k \in \mathcal{K}, \forall n \in \mathcal{N}$, and $\forall j \in \mathcal{J}$ is shown below.

$$
\bar{R}_{k n}^{j}=\log \left(1+\frac{\left(p^{j}+\delta\right) g_{k n}}{\sum_{\substack{h \in \mathcal{K} \backslash\{k\}: \\ b_{n}(h)<b_{n}(k)}}\left(\sum_{j^{\prime} \in \mathcal{J}} x_{h n}^{j^{\prime}} p^{j^{\prime}}-\delta x_{h n}^{j^{\prime}}\right) g_{k n}+\eta}\right)
$$


Recall that $\delta$ is the step in power discretization. In comparison to $L(\boldsymbol{x}, \boldsymbol{p}, \boldsymbol{\lambda})$ in $P_{\mathrm{LR}}$ and $L_{D}(\boldsymbol{x}, \boldsymbol{\lambda})$ in $P_{\mathrm{LR}-\mathrm{D}}$, the construction of $P_{\text {LR-D }}^{\prime}$ contains addition or subtraction of power $\delta$, for the purpose of ensuring that the outcome is a valid upper bound to the global optimum. This is achieved by using $\delta$ to obtain more optimistic values in all three parts of the function. In (16), for example, one power step $\delta$ is added to the signal of interest in the numerator and each interfering signal becomes weaker due to the subtraction of $\delta$ in the denominator. As a result, the overall utility for $L_{D}^{\prime}(\boldsymbol{x}, \boldsymbol{\lambda}, \mu)$ is guaranteed to be an over-estimation. From Lines 16 to 19 , bisection search is applied to obtain the optimal $\mu^{*}$ such that $z_{D}\left(\boldsymbol{\lambda}, \mu^{*}\right)=$ $\min \max L_{D}^{\prime}(\boldsymbol{x}, \boldsymbol{\lambda}, \mu)$. Then, the upper bound is delivered in $\mu \geq 0 \quad x$
$V_{\mathrm{UB}}$ in Line 20 . The validity of this upper bound is proved below.

Theorem 11. $V_{U B} \geq z^{\dagger}$.

Proof: Suppose $\boldsymbol{\lambda}^{*}$ is the multiplier vector for $P_{\mathrm{LR}-\mathrm{D}}$ when Algorithm 4 terminates. Note that $\boldsymbol{\lambda}^{*}$ for $z\left(\boldsymbol{\lambda}^{*}\right)$ may not necessarily lead to the minimum dual value $z^{*}$ in $P_{\mathrm{LR}}$, so we have $z\left(\boldsymbol{\lambda}^{*}\right) \geq z^{*} \geq z^{\dagger}$. We prove that $z_{D}\left(\boldsymbol{\lambda}^{*}, \mu^{*}\right) \geq z\left(\boldsymbol{\lambda}^{*}\right)$ holds, to show $V_{\mathrm{UB}}$ is an upper bound of $z^{\dagger}$. We use vector $\boldsymbol{p}_{c} \succ 0$ to denote the optimal power allocation for $z\left(\boldsymbol{\lambda}^{*}\right)$. Based on $\boldsymbol{p}_{c}$, we now construct a power vector $\boldsymbol{p}_{d} \succ 0$ for $P_{\text {LR-D }}^{\prime}$. Each power value in $\boldsymbol{p}_{c}$ is rounded to $\boldsymbol{p}_{d}$ such that each element in $\boldsymbol{p}_{d}$ is represented by the closest power level $j \in \mathcal{J}$, i.e., $\boldsymbol{p}_{c}+\boldsymbol{\theta}=\boldsymbol{p}_{d}$, where $|\boldsymbol{\theta}| \preceq \delta$. Given $\boldsymbol{p}_{c}$ and $\boldsymbol{p}_{d}$, the corresponding $x$-vectors $\boldsymbol{x}_{c}$ and $\boldsymbol{x}_{d}$ are derived for $P_{\mathrm{LR}}$ and $P_{\mathrm{LR}-\mathrm{D}}^{\prime}$, respectively. Substituting $\boldsymbol{\lambda}^{*}, \mu$ and $\boldsymbol{x}_{d}$ in the objective of $P_{\mathrm{LR}-\mathrm{D}}^{\prime}$, we have $L_{D}^{\prime}\left(\boldsymbol{x}_{d}, \boldsymbol{\lambda}^{*}, \mu\right)$. Since we over-calculate the objective in $P_{\text {LR-D }}^{\prime}$, the summation of part I and part II in $L_{D}^{\prime}\left(\boldsymbol{x}_{d}, \boldsymbol{\lambda}^{*}, \mu\right)$ is greater than or equal to $L\left(\boldsymbol{x}_{c}, \boldsymbol{p}_{c}, \boldsymbol{\lambda}^{*}\right)$. Part III is no less than zero in $L_{D}^{\prime}\left(\boldsymbol{x}_{d}, \boldsymbol{\lambda}^{*}, \mu\right)$ for any $\mu \geq 0$ due to $\left\|\boldsymbol{p}_{c}\right\|_{1} \leq P_{\text {tot }}$ and $|\boldsymbol{\theta}| \preceq \delta$. Then $L_{D}^{\prime}\left(\boldsymbol{x}_{d}, \boldsymbol{\lambda}^{*}, \mu\right) \geq L\left(\boldsymbol{x}_{c}, \boldsymbol{p}_{c}, \boldsymbol{\lambda}^{*}\right)=z\left(\boldsymbol{\lambda}^{*}\right)$ for any $\mu \geq 0$. Thus, we have $L_{D}^{\prime}\left(\boldsymbol{x}_{d}, \boldsymbol{\lambda}^{*}, \mu^{*}\right) \geq z\left(\boldsymbol{\lambda}^{*}\right)$. Note that for $\lambda^{*}$ and $\mu^{*}$, $V_{\mathrm{UB}}=z_{D}\left(\boldsymbol{\lambda}^{*}, \mu^{*}\right)$ is the optimum value of the Lagrangian relaxation in $P_{\mathrm{LR}-\mathrm{D}}^{\prime}$, whereas $L_{D}^{\prime}\left(\boldsymbol{x}_{d}, \boldsymbol{\lambda}^{*}, \mu^{*}\right)$ is not because $\boldsymbol{x}_{\boldsymbol{d}}$ is not necessarily an optimal power allocation. Therefore, $z_{D}\left(\boldsymbol{\lambda}^{*}, \mu^{*}\right) \geq L_{D}^{\prime}\left(\boldsymbol{x}_{d}, \boldsymbol{\lambda}^{*}, \mu^{*}\right)$, and the conclusion follows.

On the complexity of LDDP, we observe the following. Within each iteration, N-LDDP calls Algorithm TSDP that has polynomial-time complexity $\mathcal{O}\left(K N M J^{2}\right)$ and hence is scalable. Note that the number of power levels $J$ can be tuned from the complexity perspective. An iteration of N-LDDP may require the conversion to feasible power allocation (Line 9). It is easily observed that this three-step conversion, as outlined earlier, has a complexity of $\mathcal{O}\left(K N \log _{2}(K N)\right)$, which scales much better than $\mathcal{O}\left(K N M J^{2}\right)$. Next, obtaining the upper bound in UB-LDDP consists of using Algorithm TSDP in one-dimensional bi-section search. This computation does not lead to the computational bottleneck, because the upper bound is computed only once, and its purpose is not for power allocation in NOMA, but for performance evaluation as a post-processing step. Hence, overall, the complexity is determined by N-LDDP, and equals $\mathcal{O}\left(C K N M J^{2}\right)$, where $C$ is the number of subgradient optimization iterations upon termination. Subgradient optimization for Lagrangian duality has asymptotic convergence in general. In the next section, however, we observe that convergence is approached with only a few iterations.

\section{ViI. Performance Evaluation}

\section{A. Experimental Setup}

We have carried out performance studies in downlink with randomly and uniformly distributed users. Table I summarizes the key parameters. We generate one hundred instances and consider the average performance. To evaluate the performance of LDDP, we have implemented a previous NOMA power and channel allocation scheme called "fractional transmit power control" (NOMA-FTPC) and an OFDMA scheme with FTPC (OFDMA-FTPC) [10]. In these two schemes, the set of multiplexed users $\mathcal{U}_{n}$ for each subcarrier $n$ is determined by a greed-based user grouping strategy, where $\left|\mathcal{U}_{n}\right|=M$ for NOMA-FTPC and $\left|\mathcal{U}_{n}\right|=1$ for OFDMA-FTPC. Based on the user allocation, the FTPC method is then used for power allocation. In FTPC, more power is allocated to the users with inferior channel condition for the fairness consideration [10].

Table I

Simulation PARAMETERS.

\begin{tabular}{ll}
\hline Parameter & Value \\
Cell radius & $200 \mathrm{~m}$ \\
Carrier frequency & $2 \mathrm{GHz}$ \\
Total bandwidth $(B)$ & $4.5 \mathrm{MHz}$ \\
Number of subcarriers $(N)$ & 5 in NOMA, 25 in OFDMA \\
Number of users $(K)$ & 4 to 20 \\
Path loss & COST-231-HATA \\
Shadowing & Log-normal, $8 \mathrm{~dB}$ standard deviation \\
Fading & Rayleigh flat fading [25] \\
Noise power spectral density & $-173 \mathrm{dBm} / \mathrm{Hz}$ \\
Total power $\left(P_{t o t}\right)$ & $1 \mathrm{~W}$ \\
Number of power levels $(J)$ & 20 to 100 \\
Minimum power unit $\left(\delta=\frac{P_{t o t}}{J}\right)$ & 0.01 to $0.05 \mathrm{~W}$ \\
User power limit $\left(P_{k}\right)$ & $0.2 \mathrm{~W}$ \\
Parameter $M$ & 2 to 6 \\
Tolerance $\epsilon$ in LDDP & $10^{-5}$ \\
$C_{\text {max }}$ in LDDP & 200 \\
\hline
\end{tabular}

For OFDMA-FTPC, following the LTE standard, the overall bandwidth of $4.5 \mathrm{MHz}$ is divided into twenty-five subchannels with the bandwidth of $180 \mathrm{kHz}$ for each. For NOMA implementation, considering the fact that the decoding complexity and signaling overhead increase with the number of subcarriers [6], and following the NOMA setup in [10], we consider five subcarriers with the bandwidth of $900 \mathrm{kHz}$ for each in NOMAFTPC and in the proposed LDDP.

In the simulations, N-LDDP aims to deliver a near-optimal solution (also a lower bound), and UB-LDDP, by design, provides an upper bound for global optimum. In the following, we examine five performance aspects. First, a comparative study for the SR utility of LDDP, NOMA-FTPC and OFDMAFTPC is carried out. Second, we evaluate the convergence behavior of LDDP. Third, we consider the WSR utility and examine users' fairness. Fourth, we evaluate the throughput performance for the cell-edge users. Fifth, we investigate the characteristics of user grouping in N-LDDP. 


\section{B. Performance in Throughput and Bounding}

Applying the three algorithms to all instance, the average results are summarized in Fig. 1 to 3. In Fig. 1, we evaluate the SR utility with respect to user number $K$, with setting $M=2$ and $J=100$. We make the following observations. First, the performance improvement tends to be marginal for larger $K$ in all the schemes. This is expected since the multiuser diversity is effective when the number of users is small, and is saturated if $K$ is large. Second, N-LDDP outperforms NOMAFTPC and OFDMA-FTPC. N-LDDP achieves performance improvement of around 20\% over NOMA-FTPC. NOMAFTPC, in turn, performs much better than OFDMA. Third, N-LDDP is capable of providing near-optimal solutions. The average gap between UB-LDDP and N-LDDP is $11 \%$ in average, and the variation of the gap is insensitive to the number of users. This implies that the gap between N-LDDP and the global optimum $z^{\dagger}$ is even smaller than $11 \%$.

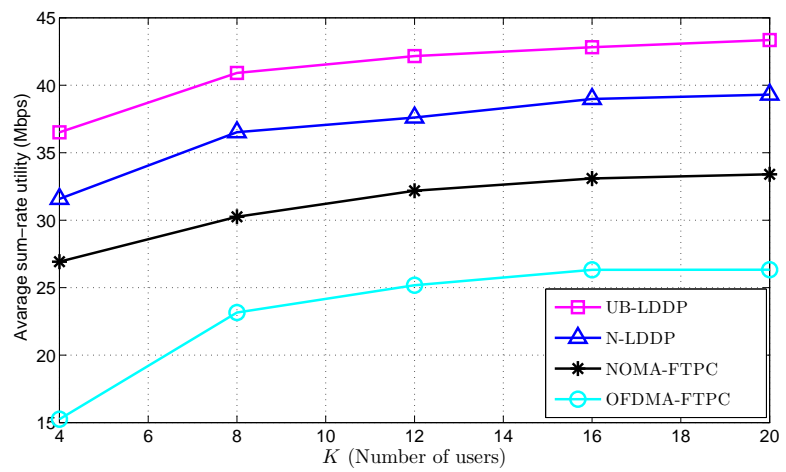

Figure 1. LDDP performance in respect of the number of users.

Next, the impact of parameters $J$ and $M$ is evaluated. The instances with $K=20$ are used in the simulations, and $M=2$ in Fig. 2 and $J=100$ in Fig. 3. From Fig. 2, increasing $J$ leads to progressively tighter intervals between UB-LDDP and N-LDDP since larger $J$ provides better granularity in power discretization, and thus improves the solution quality. Moreover, we observe that the improvement comes mainly from UB-LDDP. This is because, for delivering the upper bound, the objective value in $P_{\mathrm{LR}-\mathrm{D}}^{\prime}$ has been intentionally over-calculated by $\delta=\frac{P_{t o t}}{J}$. Compared to using $\delta=0$, applying $\delta>0$ in the over-calculation results in an excess of utility in the objective. This excess part is clearly $J$-related, and is significantly reduced when $J$ is large.

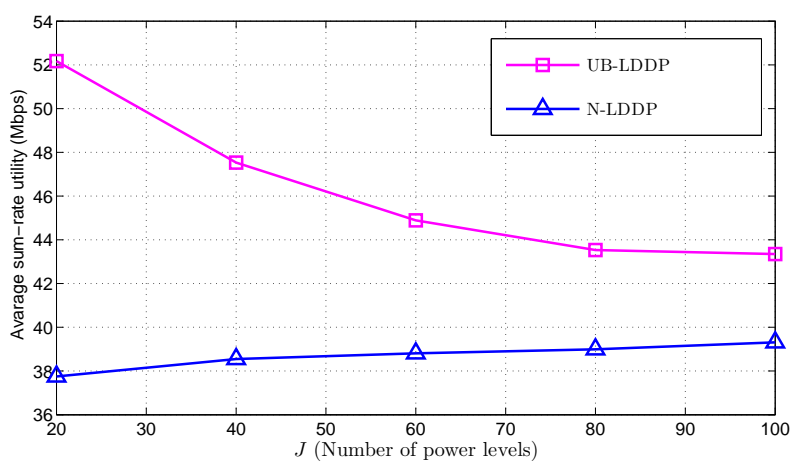

Figure 2. LDDP bounding performance in respect of parameter $J$.

In Fig. 3, more users are allowed to share the same subcarrier. Increasing $M$ leads to more total throughput for both
NOMA schemes. One can observe that N-LDDP constantly outperforms NOMA-FTPC. We also notice that increasing $M$ results in degradation of UB-LDDP. The main reason is that when $M$ grows, the over-calculations in the objective of $P_{\text {LR-D }}^{\prime}$ are accumulated over all multiplexed users.

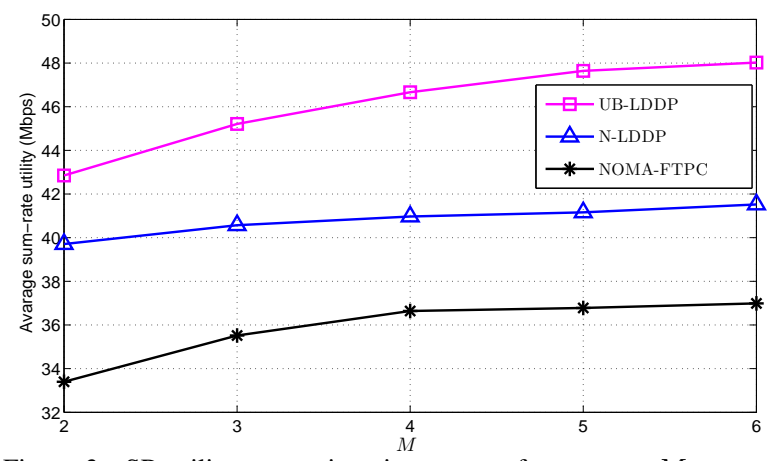

Figure 3. SR utility comparison in respect of parameter $M$.

\section{Performance in Convergence}

We illustrate the convergence behavior of LDDP by two representative instances with 4 and 20 users, respectively, with $N=5, J=100$, and $M=2$. The evolution of the values of $z_{D}(\boldsymbol{\lambda})$ and $V_{\mathrm{LB}}$ over the iteration number $C$ is provided in Fig. 4.

From the figure, a majority of the iterations is part of the tailing-off effect. The utility ( $V_{\mathrm{LB}}$ of the algorithm) and the Lagrangian dual function $\left(z_{D}(\boldsymbol{\lambda})\right)$ both approach the achievable values with 10 iterations or fewer. Note that each iteration has polynomial-time complexity, see Theorem 10.

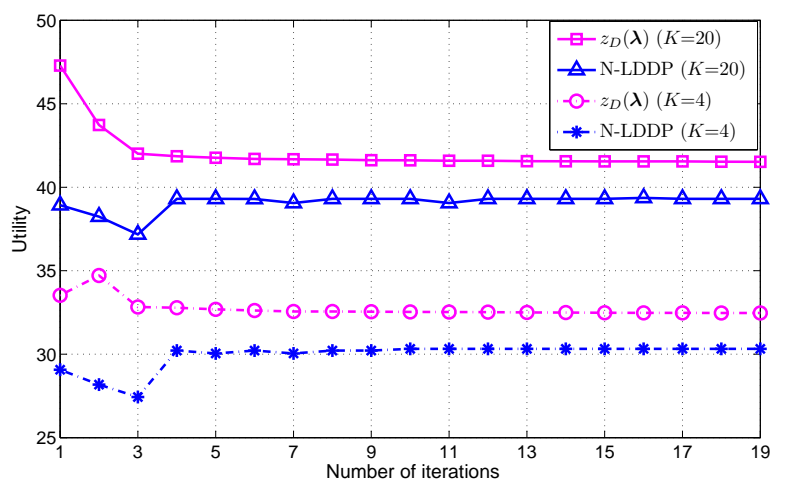

Figure 4. Illustration for LDDP convergence with $K=4$ and 20, $N=5$, $J=100$, and $M=2$.

\section{Performance in Fairness}

As our next part of results, we evaluate the performance for LDDP, NOMA-FTPC, and OFDMA-FTPC in fairness. We examine the fairness over a scheduling time period. As was mentioned earlier, scheduling in the time domain is slotted. Denote by $t$ the time slot index. We define a scheduling frame consisting of 20 slots. The channel state information is collected once per frame. Define $\bar{R}_{k}(t)=\left(1-\frac{1}{T}\right) \bar{R}_{k}(t-$ 1) $+\frac{1}{T} r_{k}(t-1)$ as user $k$ 's average rate prior to slot $t$, where parameter $T$ is the length of a time window (in the number of time slots), and $r_{k}(t-1)$ is user $k$ 's instantaneous rate in slot $t-1$ [15]. By proportional fairness, the weight of user $k$ in slot $t$ is set to $1 / \bar{R}_{k}(t)$, and for each slot, N-LDDP, NOMAFTPC, and OFDMA-FTPC with WSR utility maximization are performed once. 
Suppose the average users' rates are $\bar{R}_{1}, \ldots, \bar{R}_{K}$ at the end of the scheduling period, and consider the Jain's fairness index, computed as $\frac{\left(\sum_{k=1}^{K} \bar{R}_{k}\right)^{2}}{K \sum_{k=1}^{K} \bar{R}_{k}^{2}}$. This index, developed in [32], is widely used as a fairness measure for user throughput in communications networks. The value of this fairness index is between $\frac{1}{K}$ and 1.0. A higher value indicates fairer throughput distribution, and the maximum value of 1.0 is reached if and only if all users achieve exactly the same throughput. Note that the use of this index, by itself, does not prevent a user from being served with low throughput (or even zero throughput), which, however, will most likely bring down the value of the index. In our case, zero or very low throughput of any user is avoided by the fact that the weights in W-JPCAP are set in accordance with proportionally fair scheduling. Hence, over time, the weight of a user will increase to infinity, if the user keeps being allocated with zero throughput. For further insights of user throughput, particularly throughput of celledge users, please see Section VII-E.

The fairness index in respect of $K$ and $M$ is shown in Fig. 5 and Fig. 6, respectively. The parameters are set to be $M=2$ in Fig. 5 and $K=20$ in Fig. 6. For both figures, we consider a scheduling period of 100 time slots, with $J=100$ and $T=50$. From Fig. 5 and 6 , we observe that, first, the proposed N-LDDP achieves the best performance. Moreover, Fig. 5, increasing $K$ leads to fairness degradation in all schemes due to more competition among the users.

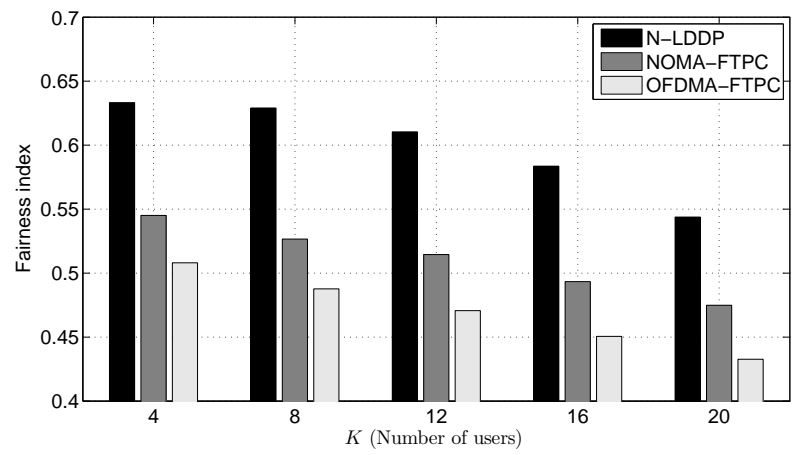

Figure 5. Fairness comparison in respect of the number of users.

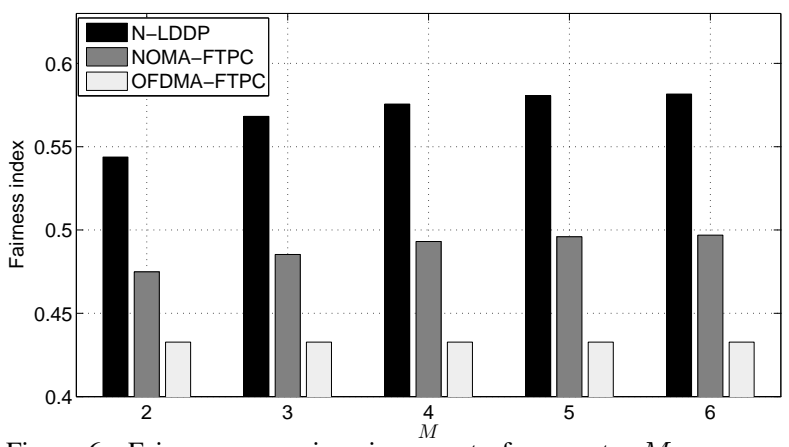

Figure 6. Fairness comparison in respect of parameter $M$.

From Fig. 6, the fairness index increases in $M$. This is because a larger $M$ provides more flexibility in resource allocation among the users. There is however a saturation effect, showing that the constraining impact due to limiting the number of multiplexed users per subcarrier decreases when the limit becomes large. Note that in Fig. 3, the improvement of throughput is marginal with $M$ becomes large. Thus a mod- erate $M$ is justified not only by implementation complexity, but also that having a large $M$ may not lead to significant performance improvement.

In the two figures, OFDMA-FTPC gives the lowest fairness index. A particular reason is that the FTPC channel and power allocation scheme is sub-optimal. This also explains the improvement enabled by the proposed power optimization algorithm in comparison to NOMA-FTPC. We also remark that the vertical axis of the two figures starts from a positive value, hence the relative difference between the schemes in fairness is smaller than what it may appear to be.

\section{E. Performance for Cell-edge Users in Throughput}

The performance of cell-edge users is of significance. To evaluate, we split the service area of the cell into an edge zone and a center zone. Performance comparison is carried out for twenty-user instances with $M=2, J=100$, and 100 time slots. In the simulations, we deploy half of the users to the cell edge in each instance. Each value in Fig. 7 represents the average user rate over the entire scheduling period. We observe that using LDDP significantly improves the rates for cell-edge users. From the results in Fig. 7, the average rates of all cell-edge users in N-LDDP are much more than those of NOMA-FTPC and OFDMA-FTPC.

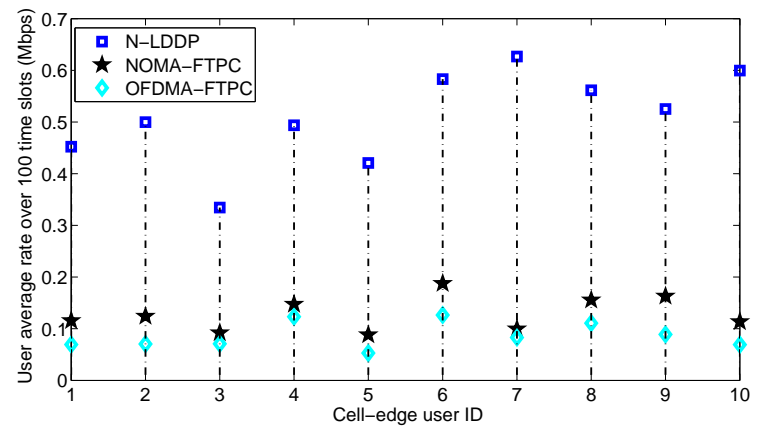

Figure 7. Performance comparison in cell-edge users.

\section{F. Characteristics of User Grouping}

The final part of our performance study is on characteristics of user grouping, i.e., which users tend to be multiplexed together on the same subcarrier by optimization, We consider a scenario with $M=2, J=100, K=20$, and apply N-LDDP to 1,000 realizations. For each subcarrier, we index the users in descending order of channel gain. If there are two users multiplexed on the subcarrier in the algorithm solution, we consider the difference of the two user indices. For example, if the users with the highest and lowest gains are grouped together, the difference is 19 . The results are illustrated in Fig. 8, where the horizontal axis is the difference in index.

From the figure, we observe that users having large difference in channel gain are more likely to be multiplexed on the same subcarrier. This is coherent with the conclusion in [18]. On the other hand, it is also evident by the figure that optimal assignment is not necessarily to select users with the best and poorest gains on a subcarrier. The observation motivates the treatment of subcarrier allocation as optimization variables. 


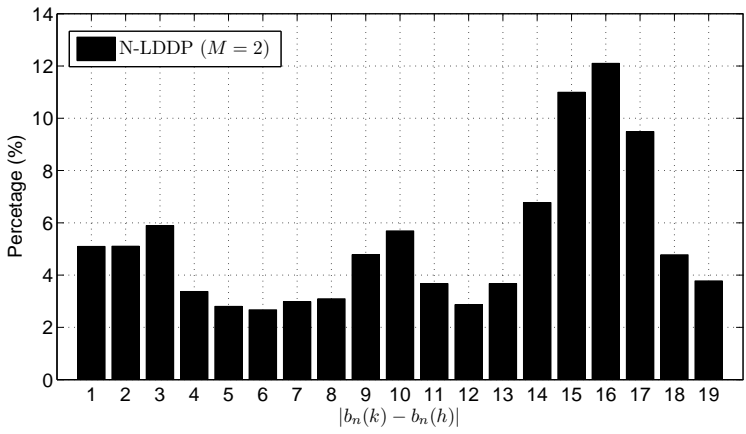

Figure 8. The results of user grouping in N-LDDP.

\section{CONCLUSIONS}

We have considered jointly optimizing power and channel allocation for NOMA. Theoretical insights on complexity and optimality have been provided, and we have proposed an algorithm framework based on Lagrangian dual optimization and dynamic programming. The proposed algorithm is capable of providing near-optimal solutions as well as bounding the global optimum tightly. Numerical results demonstrate that the proposed algorithmic notions result in significant improvement of throughput and fairness in comparison to existing OFDMA and NOMA schemes.

An extension of the work is the consideration of maxmin fairness for one scheduling instance. In this case, one solution approach is to perform a bi-section search. For each target level that represents the minimum throughput required for all users, the problem reduces to a feasibility test, i.e., whether or not the target is achievable subject to the power limits. This can be formulated as to minimize the total power, with constraints specifying the throughput target value and user-individual power limits. The development of optimization algorithms for this problem is subject to further study.

\section{ACKNOWLEDGMENTS}

We would like to thank the anonymous reviewers for their comments and suggestions. This work has been supported by the European Union Marie Curie project MESH-WISE (324515), Career LTE (329313). The work of the first author has been supported by the China Scholarship Council (CSC).

\section{REFERENCES}

[1] D. Yuan, J. Joung, C. K. Ho, and S. Sun, "On tractability aspects of optimal resource allocation in OFDMA systems," IEEE Transactions on Vehicular Technology, vol. 62, no. 2, pp. 863-873, Feb. 2013.

[2] L. Lei, D. Yuan, C. K. Ho, and S. Sun, "A unified graph labeling algorithm for consecutive-block channel allocation in SC-FDMA," IEEE Transactions on Wireless Communications, vol. 12, no. 11, pp. 57675779, Nov. 2013.

[3] J. Andrews, S. Buzzi, W. Choi, S. Hanly, A. Lozano, A. Soong, and J. Zhang, "What will 5G be?" IEEE Journal on Selected Areas in Communications, vol. 32, no. 6, pp. 1065-1082, June 2014.

[4] Q. Li, H. Niu, A. Papathanassiou, and G. Wu, "5G network capacity: Key elements and technologies," IEEE Vehicular Technology Magazine, vol. 9, no. 1, pp. 71-78, Mar. 2014.

[5] W. Nam, D. Bai, J. Lee, and I. Kang, "Advanced interference management for 5G cellular networks," IEEE Communications Magazine, vol. 52, no. 5, pp. 52-60, May 2014.

[6] K. Higuchi and A. Benjebbour, "Non-orthogonal multiple access (NOMA) with successive interference cancellation for future radio access," IEICE Transactions on Communications, vol. 98, no. 3, pp. 403-414, Mar. 2015.
[7] N. Jindal, S. Vishwanath, and A. Goldsmith, "On the duality of Gaussian multiple-access and broadcast channels," IEEE Transactions on Information Theory, vol. 50, no. 5, pp. 768-783, May 2004.

[8] J. Andrews, "Interference cancellation for cellular systems: a contemporary overview," IEEE Wireless Communications, vol. 12, no. 2, pp. 19-29, Apr. 2005.

[9] P. Xu, Z. Ding, X. Dai, and H. Poor, "NOMA: An information theoretic perspective," 2015. [Online]. Available: http://arxiv.org/abs/1504.07751

[10] Y. Saito, A. Benjebbour, Y. Kishiyama, and T. Nakamura, "System-level performance evaluation of downlink non-orthogonal multiple access (NOMA)," in IEEE PIMRC, Sept. 2013, pp. 611-615.

[11] A. Benjebbour, Y. Saito, Y. Kishiyama, A. Li, A. Harada, and T. Nakamura. "Concept and practical considerations of non-orthogonal multiple access (NOMA) for future radio access," in IEEE ISPACS, Nov. 2013.

[12] L. Lei, D. Yuan, C. K. Ho, and S. Sun, "Joint optimization of power and channel allocation with non-orthogonal multiple access for $5 \mathrm{G}$ cellular systems," in IEEE GLOBECOM, Dec. 2015, pp. 1-6.

[13] Z. Ding, Z. Yang, P. Fan, and H. Poor, "On the performance of nonorthogonal multiple access in $5 \mathrm{G}$ systems with randomly deployed users," IEEE Signal Processing Letters, vol. 21, no. 12, pp. 1501-1505, Dec. 2014.

[14] S. Timotheou and I. Krikidis, "Fairness for non-orthogonal multiple access in 5G systems," IEEE Signal Processing Letters, vol. 22, no. 10, pp. 1647-1651, Oct. 2015.

[15] M. Mollanoori and M. Ghaderi, "Uplink scheduling in wireless networks with successive interference cancellation," IEEE Transactions on Mobile Computing, vol. 13, no. 5, pp. 1132-1144, May 2014.

[16] Y. Endo, Y. Kishiyama, and K. Higuchi, "Uplink non-orthogonal access with MMSE-SIC in the presence of inter-cell interference," in IEEE ISWCS, Aug. 2012, pp. 261-265.

[17] M. Al-Imari, P. Xiao, M. Imran, and R. Tafazolli, "Uplink nonorthogonal multiple access for 5G wireless networks," in IEEE ISWCS, Aug. 2014, pp. 781-785.

[18] Z. Ding, P. Fan, and H. V. Poor, "Impact of user pairing on 5G nonorthogonal multiple access downlink transmissions," IEEE Transactions on Vehicular Technology, vol. PP, no. 99, pp. 1-1, Sept. 2015.

[19] B. Di, S. Bayat, L. Song, and Y.Li, "Radio resource allocation for downlink non-orthogonal multiple access (NOMA) networks using matching theory," in IEEE GLOBECOM, Dec. 2015.

[20] Y. Sun, D. Ng, Z. Ding, and R. Schober, "Optimal joint power and subcarrier allocation for MC-NOMA systems,", in IEEE GLOBECOM, Dec. 2016 (to appear, arXiv:1603.08132).

[21] R. Etkin, D. Tse, and H. Wang, "Gaussian interference channel capacity to within one bit," IEEE Transactions on Vehicular Technology, vol. 54, no. 12, pp. 5534-5562, 2008.

[22] Y. Liu and Y. Dai, "On the complexity of joint subcarrier and power allocation for multi-user OFDMA systems," IEEE Transactions on Signal Processing, vol. 62, no. 3, pp. 583-596, Feb. 2014.

[23] S. Hayashi and Z. Luo, "Spectrum management for interference-limited multiuser communication systems," IEEE Transactions on Information Theory, vol. 55, no. 3, pp. 1153-1175, Mar. 2009.

[24] Z. Luo and S. Zhang, "Dynamic spectrum management: Complexity and duality," IEEE Journal of Selected Topics in Signal Processing, vol. 2, no. 1, pp. 57-73, Feb. 2008.

[25] D. Tse and P. Viswanath, Fundamentals of Wireless Communication. Cambridge University Press, 2005.

[26] K. Seong, M. Mohseni, and J. Cioffi, "Optimal resource allocation for OFDMA downlink systems," in IEEE ISIT, July 2006, pp. 1394-1398.

[27] K. Seong, D. Yu, Y. Kim, and J. Cioffi, "Optimal resource allocation via geometric programming for OFDM broadcast and multiple access channels," in IEEE GLOBECOM, Nov. 2006, pp. 1-5.

[28] S. Boyd and L. Vandenberghe, Convex Optimization. Cambridge, UK: Cambridge University Press, 2004.

[29] ETSI, "Evolved universal terrestrial radio access (E-UTRA) and evolved universal terrestrial radio access network (E-UTRAN); overall description; stage 2 (3GPP TS 36.300 version 10.5.0 Release 10)," ETSI TS 136300 V10.5.0, Nov. 2011.

[30] R. K. Ahuja, T. L. Magnanti, and J. B. Orlin, Network Flows: Theory, Algorithms, and Applications. Prentice-Hall, 1993.

[31] H. Kellerer, U. Pferschy, and D. Pisinger, Knapsack Problems. Springer, 2004.

[32] R. Jain, D. Chiu, and W. Hawe, "A quantitative measure of fairness and discrimination for resource allocation in shared computer systems," Eastern Research Lab, Digital Equipment Corporation, DEC Technical Report 301, Sept. 1984. 


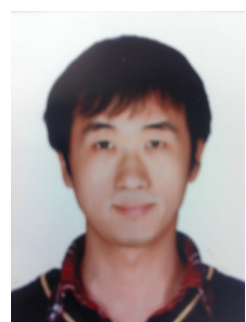

Lei Lei received the B.Eng. and M.Eng. degrees from Northwestern Polytechnical University, Xi'an, China, in 2008 and 2011, respectively. He obtained his Ph.D. degree in 2016 at the Department of Science and Technology, Linköping University, Sweden. $\mathrm{He}$ is a research associate at the Interdisciplinary Centre for Security, Reliability and Trust (SnT), University of Luxembourg. He was a research assistant at Institute for Infocomm Research $\left(\mathrm{I}^{2} \mathrm{R}\right)$, A*STAR, Singapore, from June 2013 to December 2013. He received the IEEE Sweden Vehicular Technology-Communications-Information Theory (VT-COM-IT) joint chapter best student journal paper award in 2014. His current research interests include resource allocation and optimization in $4 \mathrm{G}$ and $5 \mathrm{G}$ wireless networks, and energy-efficient communications.

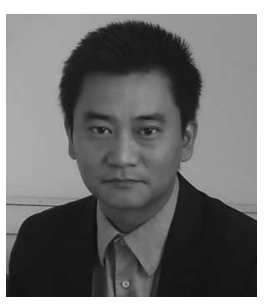

Di Yuan received his MSc degree in Computer Science and Engineering, and $\mathrm{PhD}$ degree in Optimization at Linköping Institute of Technology in 1996 and 2001, respectively. He is full professor in telecommunications at the Department of Science and Technology, Linköping University, and head of a research group in mobile telecommunications. At present he is Visiting Professor at University of Maryland, College Park, MD, USA. His current research mainly addresses network optimization of $4 \mathrm{G}$ and $5 \mathrm{G}$ systems, and capacity optimization of wireless networks. Dr. Yuan has been guest professor at the Technical University of Milan (Politecnico di Milano), Italy, in 2008, and senior visiting scientist at Ranplan Wireless Network Design Ltd, United Kingdom, in 2009 and 2012. In 2011 and 2013 he has been part time with Ericsson Research, Sweden. He is an area editor of the Computer Networks journal. He has been in the management committee of four European Cooperation in field of Scientific and Technical Research (COST) actions, invited lecturer of European Network of Excellence EuroNF, and Principal Investigator of several European FP7 and Horizon 2020 projects. He is a co-recipient of IEEE ICC' 12 Best Paper Award, and supervisor of the Best Student Journal Paper Award by the IEEE Sweden Joint VT-COM-IT Chapter in 2014. Dr. Yuan is a Senior Member of IEEE.

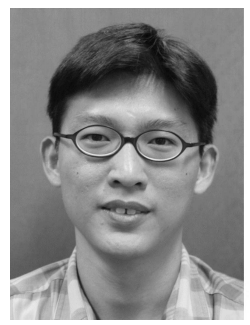

Chin Keong Ho received the B. Eng. (First-Class Hons., Minor in Business Admin.), and M. Eng degrees from the Department of Electrical Engineering, National University of Singapore in 1999 and 2001, respectively. He obtained his Ph.D. degree in 2009 at the Eindhoven University of Technology, The Netherlands, where he concurrently conducted research work in Philips Research. Since August 2000 he has been with Institute for Infocomm Research (I2R), A*STAR, Singapore. His research interest includes green wireless communications with focus on energy-efficient solutions and with energy harvesting constraints; cooperative and adaptive wireless communications; implementation aspects of multicarrier and multi-antenna communications; innovative use of communications in the field of robotics. His works received the Best Student Journal Paper Award of IEEE Sweden VT-COM-IT Joint Chapter in 2014, the IEEE Marconi Prize Paper Award in 2015, and the IEEE ComSoc Asia-Pacific Outstanding Paper Award in 2016.

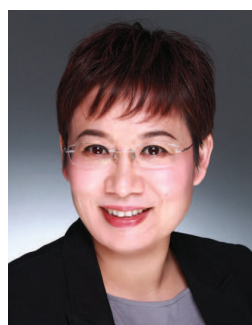

Sumei Sun is Head of the Communications and Networks Cluster, Institute for Infocomm Research, Agency for Science, Technology, and Research, Singapore. Her research focus is energy- and spectrumefficient communication technologies for connecting human, machines, and things. Dr Sun is inventor and co-inventor of thirty granted patents and more than thirty pending patent applications, many of which have been licensed to industry. She has authored and co-authored more than two hundred technical papers in prestigious IEEE journals and conferences. She has also been actively contributing to organizing conferences in different roles. Some of her recent conference services include Executive Vice Chair of Globecom 2017, Symposium Co-Chair of ICC 2015 and 2016, Track Co-Chair of IEEE VTC 2012 Spring, VTC 2014 Spring and VTC 2016 Fall, Publicity Co-Chair of PIMRC 2015, etc. She is an Editor for IEEE Transactions on Vehicular Technology (TVT) since 2011, Editor for IEEE Wireless Communication Letters during 2011-2016, and Editor of IEEE Communications Surveys and Tutorials since 2015. She received the "Top Editor Award" in 2016, "Top15 Outstanding Editors" recognition in 2014, and Top Associate Editor recognition in 2013 and 2012, all from TVT. She is a distinguished lecturer of IEEE Vehicular Technology Society 2014-2018, a co-recipient of the 16th PIMRC Best Paper Award, and Distinguished Visiting Fellow of the Royal Academy of Engineering, UK, in 2014. She is a Fellow of the IEEE, class 2016. 